\title{
Ezh2 loss propagates hypermethylation at T cell differentiation-regulating genes to promote leukemic transformation
}

\author{
Changshan Wang, ${ }^{1,2}$ Motohiko Oshima, ${ }^{2}$ Daisuke Sato, ${ }^{2}$ Hirotaka Matsui, ${ }^{3}$ Sho Kubota, ${ }^{4}$ Kazumasa Aoyama, ${ }^{2}$ \\ Yaeko Nakajima-Takagi, ${ }^{2}$ Shuhei Koide, ${ }^{2}$ Jun Matsubayashi, ${ }^{5}$ Makiko Mochizuki-Kashio, ${ }^{2}$ Takako Nakano-Yokomizo, ${ }^{4}$ \\ Jie Bai, ${ }^{4}$ Toshitaka Nagao, ${ }^{5}$ Akinori Kanai, ${ }^{6}$ Atsushi Iwama, ${ }^{2,7}$ and Goro Sashidaa ${ }^{2,4}$ \\ 'The State Key Laboratory of Reproductive Regulation and Breeding of Grassland Livestock, School of Life Sciences, Inner Mongolia University, Hohhot, China. ²Department of Cellular and Molecular \\ Medicine, Graduate School of Medicine, Chiba University, Chiba, Japan. ${ }^{3}$ Department of Molecular Laboratory Medicine, Faculty of Life Sciences, and ${ }^{4}$ Laboratory of Transcriptional Regulation in \\ Leukemogenesis, International Research Center for Medical Sciences, Kumamoto University, Kumamoto, Japan. ${ }^{5}$ Department of Anatomic Pathology, Tokyo Medical University, Tokyo, Japan. ${ }^{6}$ Department \\ of Molecular Oncology, Research Institute for Radiation Biology and Medicine, Hiroshima University, Hiroshima, Japan. 'Division of Stem Cell and Molecular Medicine, Center for Stem Cell Biology and \\ Regenerative Medicine, Institute of Medical Science, University of Tokyo, Tokyo, Japan.
}

Early T cell precursor acute lymphoblastic leukemia (ETP-ALL) is a new pathological entity with poor outcomes in T cell ALL (T-ALL) that is characterized by a high incidence of loss-of-function mutations in polycomb repressive complex 2 (PRC2) genes. We generated a mouse model of ETP-ALL by deleting Ezh2, one of the PRC2 genes, in p53-null hematopoietic cells. The loss of Ezh2 in p53-null hematopoietic cells impeded the differentiation of ETPs and eventually induced ETP-ALL-like disease in mice, indicating that PRC2 functions as a bona fide tumor suppressor in ETPs. A large portion of PRC2 target genes acquired DNA hypermethylation of their promoters following reductions in $\mathrm{H} 3 \mathrm{~K} 27$ me3 levels upon the loss of Ezh2, which included pivotal T cell differentiation-regulating genes. The reactivation of a set of regulators by a DNA-demethylating agent, but not the transduction of single regulator genes, effectively induced the differentiation of ETP-ALL cells. Thus, PRC2 protects key T cell developmental regulators from DNA hypermethylation in order to keep them primed for activation upon subsequent differentiation phases, while its insufficiency predisposes ETPs to leukemic transformation. These results revealed a previously unrecognized epigenetic switch in response to PRC2 dysfunction and provide the basis for specific rational epigenetic therapy for ETP-ALL with PRC2 insufficiency.

\section{Introduction}

Early $\mathrm{T}$ cell precursor acute lymphoblastic leukemia (ETP-ALL) has been identified as a new pathological entity of T cell ALL (T-ALL) that shows resistance to conventional chemotherapies and has a poor outcome (1). Normal ETPs are a subset of recent immigrants from $\mathrm{BM}$ to the thymus and retain the ability to differentiate into T cells and myeloid cells, but not B cells. ETP-ALL cells lack mature $\mathrm{T}$ cell-lineage markers, such as CD1a and CD8, but show the aberrant expression of surface markers for hematopoietic stem cells (HSCs) and myeloid cells (e.g., c-Kit, CD13, CD33) (1). In contrast to cortical T-ALL characterized by the activation of NOTCH1 mutations, ETP-ALL has been characterized by the activation of mutations in genes encoding cytokine receptors and RAS signaling molecules (e.g., IL7R and NRAS) as well as inactivating mutations in polycomb repressive complex 2 (PRC2) genes (2).

PRC2 consists of EZH2, EED, and SUZ12. EZH2 is an enzymatic component of PRC2 that catalyzes the trimethylation of histone $\mathrm{H} 3$ at lysine 27 (H3K27me3) and maintains the silencing of critical genes for differentiation in hematopoiesis $(3,4)$. Loss-

Conflict of interest: The authors have declared that no conflict of interest exists. Submitted: April 18, 2017; Accepted: June 19, 2018

Reference information: / Clin Invest. 2018;128(9):3872-3886.

https://doi.org/10.1172/JCI94645. of-function mutations in $E Z H 2$ were initially identified in patients with clonal myeloid malignancies originating from HSCs, such as myelodysplastic syndrome (MDS), myeloproliferative neoplasm (MPN), and MDS/MPN overlap disorders $(5,6)$. Deletions and/ or loss-of-function mutations in PRC2 genes were subsequently identified at high frequencies in patients with ETP-ALL $(16 \%$ EZH2, 17\% SUZ12, and 13\% EED) and non-ETP T-ALL patients (5\%-16\% EZH2 and 2\%-4\% SUZ12) $(2,7)$. We previously demonstrated that the hematopoietic cell-specific deletion of Ezh2 collaborated with Tet2 loss, RUNX1 mutants, or the JAK2 ${ }^{\mathrm{V} 671 F}$ mutant in the development of myeloid malignancies in mice (8-10). We and others also reported that the absence of Ezh2 alone also induced non-ETP T-ALL in mice $(11,12)$. These findings indicate that Ezh2 functions as a tumor suppressor, not only in myeloid malignancies, but also in T cell malignancies, including ETP-ALL.

In order to examine how PRC2 inactivation promotes the development of ETP-ALL in vivo, an ETP-ALL mouse model has been developed using hematopoietic progenitors deficient for Cdkn2a and Eed or Ezh2, which were transformed by NRAS ${ }^{\mathrm{Q} 1 \mathrm{~K}}$ on OP9-DLL1, a stromal cell line expressing the Notch ligand delta-like 1 (DLL1). The transformed cells induced ETP-ALL-like leukemia with a double-negative 1 thymocyte (DN1) $\left(\mathrm{CD} 44^{+} \mathrm{CD} 25^{-}\right)$ and DN2 $\left(\mathrm{CD} 44^{+} \mathrm{CD} 25^{+}\right)$surface phenotype in recipient mice (13). However, additional models that precisely recapitulate the 
phenotypic and transcriptional features of human ETP-ALL are needed in order to understand the impact of PRC2 inactivation in the pathogenesis of ETP-ALL. In the present study, we generated a mouse model of ETP-ALL by deleting Ezh2 and $p 53$ in mice. We found that Ezh2p53-deficient mice preferentially developed lethal T-ALL compatible with human ETP-ALL rather than thymic lymphoma, which typically develops in $p 53$-deficient mice (14).

We herein demonstrate how the loss of Ezh2 induced an epigenetic switch from a H3K27me3 modification to DNA hypermethylation at $\mathrm{CpG}$ island (CGI) promoter regions in thymic precursor cells, leading to the suppressed expression of critical T cell commitment regulators, such as Runx1 and $B c l 11 b$, but the sustained expression of HSC signature genes, thereby promoting the transformation of ETP-ALL. We also provide evidence for the greater sensitivity of Ezh2p53-deficient ETP-ALL cells to decitabine (DAC), a DNA-demethylating agent, which restored the differentiation of ETP-ALL cells. Our results indicate that Ezh2-PRC2 protects key T cell developmental regulator genes from DNA hypermethylation in order to keep them primed for activation upon subsequent differentiation phases; however, its disruption initiates the transformation of immature $\mathrm{T}$ cell leukemia.

\section{Results}

Ezh2 loss causes lethal hematological malignancies in the absence of p53. Since deletions and loss-of-function mutations in PRC2 genes, including $E Z H 2$, have frequently been observed in patients with ETP-ALL, we attempted to establish whether the deletion of Ezh2 promotes the development of ETP-ALL in vivo. The p53 pathway is often inactivated in patients with ETP-ALL via genetic deletions or mutations of $C D K N 2 A(2,15)$. In addition, mutations in both $p 53$ and SUZ12, a PRC2 gene, were found in a patient with ETP-ALL (16). These findings prompted us to combine PRC2 and p53 insufficiency to accelerate the development of ETP-ALL. We generated Ezh $2^{f l / f l} ; p 53^{f l / f l} ; C r e-E R T 2$ compound mice. Total BM cells isolated from Cre-ERT2, Ezh2 $2^{f / f l} ; C r e-E R T 2, p 53^{f / f l} ; C r e-E R T 2$, and $E z h 2^{f / f l} ; p 53^{f / f l} ; C r e-E R T 2$ mice were transplanted into lethally irradiated CD $45.1^{+}$WT recipient mice. We then deleted Ezh2 and/ or $p 53$ by activating Cre recombinase via intraperitoneal injections of tamoxifen at 4 weeks after transplantation (Figure 1A). We hereafter refer to recipient mice reconstituted with WT, $E z h 2^{4 / \Delta}$,

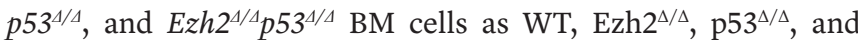
Ezh $2^{\Delta / \Delta} \mathrm{p} 53^{\Delta / \Delta}$ mice. We confirmed the successful abolishment of Ezh2 and p53 transcripts (Figure 1B) and decreased H3K27me3 levels (Figure 1C) in $\mathrm{CD}^{-} \mathrm{CD}^{-} \mathrm{DN}$ thymocytes isolated from Ezh2 ${ }^{\Delta / \Delta} \mathrm{p} 53^{\Delta / \Delta}$ mice.

Ezh2 ${ }^{\Delta / \Delta}$ mice showed leukopenia due to impaired B lymphopoiesis and variable platelet counts in peripheral blood (PB) at 3 months after transplantation (Figure 1, D and E) and developed myeloid malignancies, including MDS and MDS/MPN, but not T cell malignancies in the primary recipients (median survival, 327.5 days), as we previously reported $(8,11)$. While $\mathrm{p} 53^{\Delta / \Delta}$ mice did not show significant changes in blood cell counts at 3 months after transplantation, they died by 6 months after transplantation, with a markedly enlarged thymus due to the expansion of $\mathrm{CD}^{+} \mathrm{CD}^{+/-}$ CD8 ${ }^{+}$TCR- $\beta^{+}$tumor cells (Figure $1, \mathrm{~F}-\mathrm{H}$ ), which is compatible with thymic lymphoma, as previously reported $(14,17)$. In contrast, Ezh2 ${ }^{\Delta / \Delta} \mathrm{p} 53^{\Delta / \Delta}$ mice showed progressive anemia and severe leu- kopenia accompanied by the emergence of immature blasts in PB (Figure 1, D and E) and died by 8 months after transplantation with a longer latency than $\mathrm{p} 53^{\Delta / \Delta}$ mice (median survival, 189 days versus 137 days, $P<0.0001$ ) (Figure 1I). We observed similar survival outcomes for Ezh2 ${ }^{\Delta / \Delta} \mathrm{p} 53^{\Delta / \Delta}$ mice in 3 independent cohorts (data not shown). Taken together, the loss of Ezh2 and p53 cooperatively impaired hematopoiesis and promoted the development of a lethal hematological disease.

Ezh2 loss promotes the development of ETP-ALL in the absence of $p 53$. The accumulation of $\mathrm{CD}^{+}$single-positive (SP) cells in the thymus was evident in $\mathrm{p} 53^{\Delta / \Delta}$ mice (Figure $2, \mathrm{~A}$ and $\mathrm{B}$ ) at 3 months after transplantation, prior to the emergence of lethal disease. In contrast, Ezh2 $2^{\Delta / \Delta}$ mice and Ezh2 $2^{\Delta / \Delta} \mathrm{p} 53^{\Delta / \Delta}$ mice showed a significant increase in the proportion of $\mathrm{CD}^{-} \mathrm{CD}^{-}$(DN) T cells (Figure 2, A and B), particularly DN2 cells (Figure 2, C and D). These results indicate that the loss of Ezh2 impaired $\mathrm{T}$ cell differentiation at the DN2 to DN3 transition. Ezh2 ${ }^{\Delta / \Delta} \mathrm{p} 53^{\Delta / \Delta}$ mice eventually developed lethal disease, with a significantly enlarged thymus and spleen (Figure 2, E and F). A detailed analysis of moribund Ezh2 $2^{\Delta / \Delta}$ p53 $3^{\Delta / \Delta}$ mice revealed the expansion of leukemic blasts with a high nucleocytoplasmic ratio in the thymus in 15 out of $18 \mathrm{Ezh} 2^{\Delta / \Delta} \mathrm{p} 53^{\Delta / \Delta}$

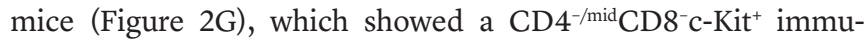
nophenotype with DN1- $\left(\mathrm{CD} 44^{+} \mathrm{CD} 25^{-}\right)$, DN2- $\left(\mathrm{CD} 44^{+} \mathrm{CD} 25^{+}\right)$, or DN1/2-like $\left(\mathrm{CD} 44^{+} \mathrm{CD} 25^{-/+}\right)$features (Figure 2, $\mathrm{H}$ and I). A flow cytometric analysis identified cytoplasmic CD3 (cyCD3) in these leukemic cells (data not shown), and a histological analysis also detected the expression of cytoplasmic CD3 (Figure 2J), consistent with the immature phenotypic hallmarks of human ETP-ALL (1). These leukemic cells massively infiltrated the spleen and liver tissues (Figure 2J) as well as PB (Figure 1E), indicating that Ezh2 ${ }^{\Delta / \Delta}$ p53 $3^{\Delta / \Delta}$ mice preferentially developed aggressive ETP-ALL. We also identified a monoclonal rearrangement of the TCR- $\beta$ loci in Ezh2 $2^{\Delta / \Delta} \mathrm{p} 53^{\Delta / \Delta}$ leukemic DN cells, indicating the thymus origin of Ezh2 $2^{\Delta / \Delta}$ p53 $3^{\Delta / \Delta}$ ETP-ALL (Figure $2 \mathrm{~K}$ ). In contrast to Ezh2 ${ }^{\Delta / \Delta}$ mice, which developed MDS and MDS/MPN after a long latency, Ezh $2^{\Delta / \Delta} \mathrm{p} 53^{\Delta / \Delta}$ mice died of ETP-ALL much earlier than Ezh2 ${ }^{\Delta / \Delta}$ mice (Figure 1I). BM analysis revealed only mild changes in the proportions of stem cells/myeloid progenitors and myeloid cells in Ezh2 $2^{\Delta / \Delta} \mathrm{p} 53^{\Delta / \Delta}$ mice (Supplemental Figure 1; supplemental material available online with this article; https://doi.org/10.1172/ JCI94645DS1), indicating that Ezh2 ${ }^{\Delta / \Delta} \mathrm{p} 53^{\Delta / \Delta}$ mice did not have apparent myeloid malignancies.

We next transplanted either BM cells or thymocyte cells (1 $\times 10^{6}$ cells) isolated from Ezh2 ${ }^{\Delta / \Delta} \mathrm{p} 53^{\Delta / \Delta}$ ETP-ALL mice into sublethally irradiated secondary recipients. All of the secondary recipients developed lethal ETP-ALL in a manner similar to the primary recipients and showed severe anemia and thrombocytopenia (Supplemental Figure 2). The secondary recipients showed obviously shorter survival than did the primary Ezh $2^{\Delta / \Delta} \mathrm{p} 53^{\Delta / \Delta}$ mice (Figure 1I and Supplemental Figure 3). Although 3 out of $18 \mathrm{Ezh} 2^{\Delta / \Delta}$ p53 ${ }^{\Delta / \Delta}$ mice developed more differentiated CD8 ${ }^{+}$TCR- $\beta^{+}$thymic lymphoma (Figure $2 \mathrm{H}$ ), similarly to $\mathrm{p} 53^{\Delta / \Delta}$ mice, these results indicate that the loss of Ezh2 facilitates the development of progressive ETP-ALL in mice lacking $p 53$.

The loss of Ezh2 impedes $T$ cell differentiation at the CD4-CD8stage. In order to understand the differentiation properties of Ezh2 $2^{\Delta / \Delta}$ $\mathrm{p} 53^{\Delta / \Delta}$ thymocytes, we cultured $\mathrm{CD} 44^{+} \mathrm{CD} 25^{-} \mathrm{DN} 1$ cells isolated 
A

Harvest $5 \times 10^{6} \mathrm{CD} 45.2^{+} \mathrm{BM}$ cells from 4 genotypes

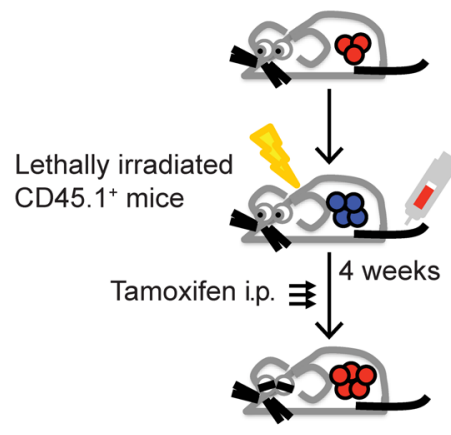

D

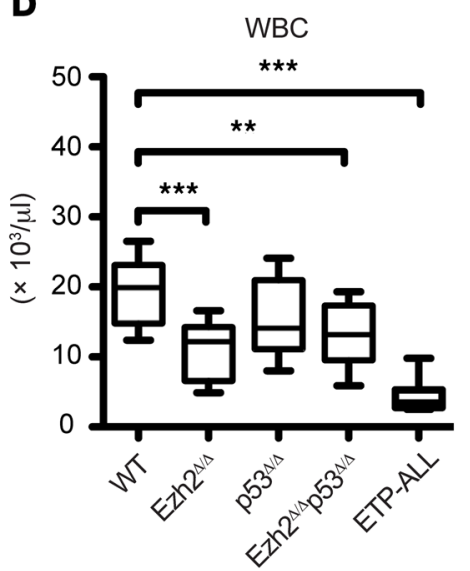

E
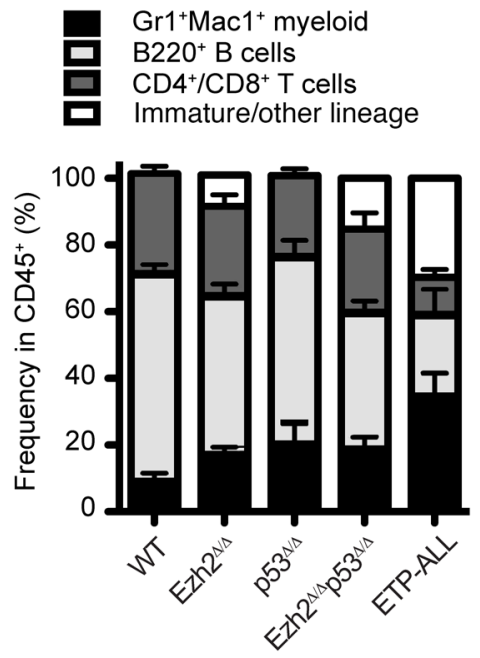

$\mathbf{F}$
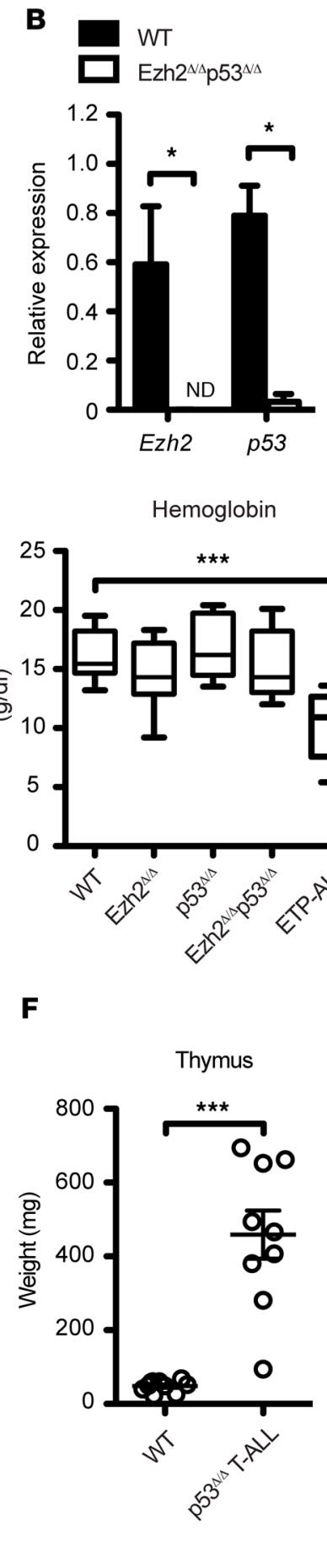

Hemoglobin
C

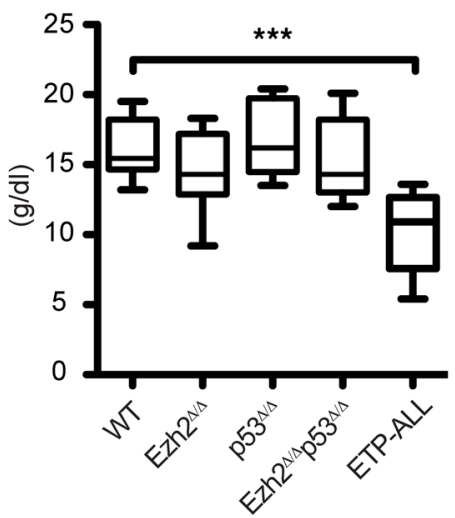

G

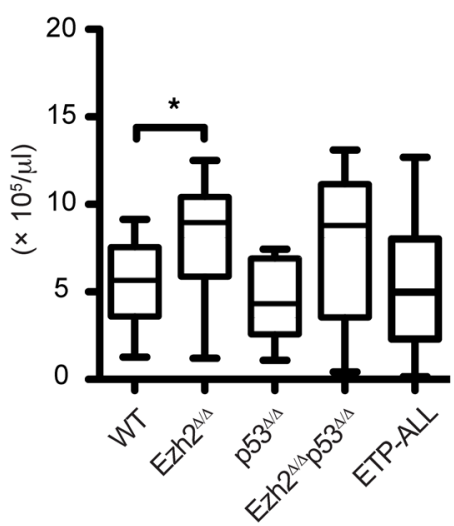

H
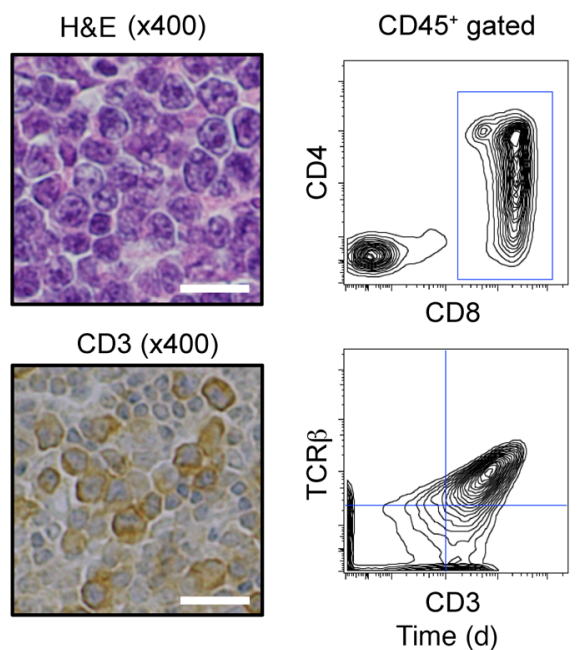

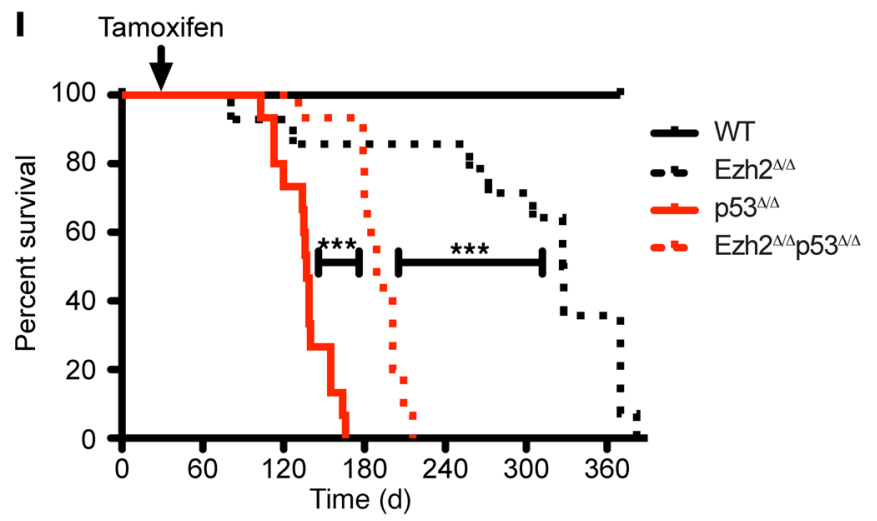


Figure 1. Ezh2 loss impaired hematopoiesis and caused lethal disease in the absence of p53. (A) Experimental schematic of our mouse model utilizing Ezh2 and/or p53 conditional knockout BM cells transplanted into lethally irradiated WT CD45.1+ recipients. (B) Quantitative RT-PCR analysis of the expression of Ezh2 and $p 53$ in CD44+CD25-CD4-CD8- (DN1) cells from WT $(n=4)$ and Ezh2 $2^{\Delta / \Delta} \mathrm{p} 53^{\Delta / \Delta}$ mice $(n=4) 4$ weeks after the deletion of Ezh2 and p53. Gapdh was used to normalize the amount of input RNA. Data are shown as mean $\pm S D$. ${ }^{*} P<0.05$, Mann-Whitney $U$ test. N.D., not determined. (C) Verification of H3K27me3 levels in CD4-CD8- (DN) cells from WT, Ezh2 $2^{\Delta / \Delta}, p 53^{\Delta / \Delta}$, and Ezh2 $2^{\Delta / \Delta} \mathrm{p} 53^{\Delta / \Delta}$ mice examined by Western blotting. Histone $\mathrm{H} 3$ was used as a loading control. (D) Complete blood cell counts of WT $(n=10)$, Ezh2 $2^{\Delta / \Delta}(n=13), \mathrm{p}^{\Delta / / \Delta}(n=15)$, and Ezh2 ${ }^{\Delta / \Delta}$ $\mathrm{p} 53^{\Delta / \Delta}(n=14)$ mice 3 months after transplantation and moribund Ezh2 $2^{\Delta / \Delta}$ p53 $3^{\Delta / \Delta}$ ETP-ALL mice $(n=11)$ at the time of sacrifice. Data are shown as box-and-whiskers plots drawing minimum to maximum. ${ }^{*} P<0.05$; ${ }^{* *} P<0.01$; ${ }^{* *} P<0.001$, Student's $t$ test. (E) Proportions of myeloid $\left(\mathrm{Gr}-1^{+}\right.$and/or Mac- $\left.{ }^{+}\right), \mathrm{B} 22 \mathrm{O}^{+} \mathrm{B}$ cells, $\mathrm{CD} 4^{+}$or $\mathrm{CD} 8^{+} \mathrm{T}$ cells, and immature cells negative for these surface markers among CD45.2+ donor-derived hematopoietic cells in PB. Data are shown as mean \pm SEM $(n=10-15)$. (F) Thymus weight of WT mice $(n=10) 3$ months after transplantation and $\mathrm{p} 53^{\Delta / \Delta} \mathrm{T}$-ALL mice $(n=9)$ at the time of sacrifice. Data are shown as mean \pm SEM. ${ }^{* *} P<0.001$, Mann-Whitney $U$ test. (C) Histology of the thymus of a p53 ${ }^{\Delta / \Delta}$ T-ALL mouse observed by H\&E staining (top) and CD3 staining (bottom). Original magnification, $\times 400$. Scale bars: $20 \mu \mathrm{m}$. (H) Representative flow cytometric profiles of $\mathrm{CD} 45^{+}$-gated thymocytes in the thymus of a $\mathrm{p} 53^{\Delta / \Delta} \mathrm{T}$-ALL mouse shown from $\mathbf{F}(n=9)$. (I) Kaplan-Meier survival curve. Median survival was significantly shorter in Ezh2 $2^{\Delta / \Delta} \mathrm{p} 53^{\Delta / \Delta}$ mice $(n=14)$ than in Ezh2 ${ }^{\Delta / \Delta}$ mice $(n=13)$ (189 days versus 327.5 days), but longer in Ezh2 $2^{\Delta / \Delta} \mathrm{p} 53^{\Delta / \Delta}$ mice $(n=14)$ than in $\mathrm{p} 53^{\Delta / \Delta}$ mice $(n=15)$ (189 days versus 137 days). ${ }^{* *} P<0.0001$, log-rank test.

from the WT, Ezh2 $2^{\Delta / \Delta}, \mathrm{p} 53^{\Delta / \Delta}$, and Ezh2 $2^{\Delta / \Delta} \mathrm{p} 53^{\Delta / \Delta}$ thymus on TSt-4/ DLL1 stromal cells, which express the Notch ligand DLL1 (Figure 3A). Ezh2 $2^{\Delta / \Delta}$ DN1 cells did not grow well (Figure 3B), presumably due to the derepression of $p 16^{\text {Inkta }}$ and $p 19^{\text {Arf }}$ in the culture (11). In contrast, Ezh $2^{\Delta / \Delta} \mathrm{p} 53^{\Delta / \Delta}$ DN1 cells showed significantly better proliferation than WT and $\mathrm{p} 53^{\Delta / \Delta}$ cells (Figure 3B), suggesting that the deletion of $p 53$ canceled the impaired growth of Ezh2 $2^{\Delta / \Delta}$ cells. While WT DN1 cells efficiently generated DP cells (Figure 3, C and D), DN1 cells deficient for $E z h 2$ showed significantly impaired differentiation and mostly stayed in the DN1 and DN2 stages, resulting in the massive expansion of Ezh $2^{\Delta / \Delta} \mathrm{p} 53^{\Delta / \Delta} \mathrm{DN} 1 / 2$ cells in the culture (Figure 3, E and F). These results match the behavior of Ezh2 $2^{\Delta / \Delta} \mathrm{p} 53^{\Delta / \Delta}$ thymocytes in vivo and indicate that the loss of both Ezh2 and p53 cooperates in the expansion of DN1/2 cells.

The loss of Ezh2 and p53 deregulates the transcriptional program of $T$ cell differentiation. In order to understand the molecular mechanisms underlying the pathogenesis of Ezh2 $2^{\Delta / \Delta} \mathrm{p} 53^{\Delta / \Delta}$ ETPALL, we performed gene expression profiling by a microarray analysis in DN1, DN2, and DN3 cells isolated from WT, Ezh2 ${ }^{\Delta / \Delta}$, $\mathrm{p} 53^{\Delta / \Delta}$, and Ezh2 $2^{\Delta / \Delta} \mathrm{p} 53^{\Delta / \Delta}$ mice at a preleukemic stage and leukemic DN2 cells isolated from 2 Ezh $2^{\Delta / \Delta} \mathrm{p} 53^{\Delta / \Delta}$ mice that developed DN1/2-type ETP-ALL. As expected, hierarchical clustering and a principal component analysis based on the microarray data revealed that the expression profiles of ETP-ALL cells were more similar to those of Ezh2 $2^{\Delta / \Delta} \mathrm{p} 53^{\Delta / \Delta}$ DN cells than those of Ezh2 $2^{\Delta / \Delta}$ and $\mathrm{p} 53^{\Delta / \Delta}$ DN cells (Figure 4, A and B). An integrative analysis revealed a set of 1,664, 2,714, and 2,210 upregulated genes and $1,432,2,516$, and 1,928 downregulated genes in Ezh2 $2^{\Delta / \Delta} \mathrm{p} 53^{\Delta / \Delta} \mathrm{DN} 2$ cells at a preleukemic stage and 2 ETP-ALL cells relative to WT DN2 cells, respectively (Figure 4C), indicating that alterations in gene expression propagated during the development of ETP-ALL. These results indicate that Ezh2 $2^{\Delta / \Delta} \mathrm{p} 53^{\Delta / \Delta}$ leukemic cells impeded the subsequent operation of $\mathrm{T}$ cell development gene networks at the DN2 and DN3 stages, but also underwent an oncogenic transcriptional reprograming that was not seen in the absence of either Ezh2 or p53.

The loss of Ezh2 leads to the silencing of critical T cell-lineage determinants. In order to clarify how the deletion of $E z h 2$ altered the transcriptional program of $\mathrm{T}$ cell development, we performed ChIP-sequencing (ChIP-seq) of H3K27me3 in DN1 cells isolated from WT, Ezh2 $2^{\Delta / \Delta}, \mathrm{p} 53^{\Delta / \Delta}$, and Ezh2 $2^{\Delta / \Delta} \mathrm{p} 53^{\Delta / \Delta}$ mice. We found that H3K27me3 levels at the promoter regions of RefSeq genes were significantly lower in Ezh2 $2^{\Delta / \Delta}$ cells and Ezh2 $2^{\Delta / \Delta} \mathrm{p} 53^{\Delta / \Delta}$ cells, but not in $\mathrm{p} 53^{\mathrm{s} / \Delta}$ cells, than in WT cells (Figure 5A). A gene set enrichment analysis (GSEA) revealed that the canonical PRC2 target genes, which were defined by the levels of H3K27me3 in WT DN1 cells ( $\geq 2.5$-fold enrichment over the input signal) (Supplemental Table 1), were positively enriched in Ezh $2^{\Delta / \Delta} \mathrm{DN} 2$ cells and Ezh $2^{\Delta / \Delta} \mathrm{p} 53^{\Delta / \Delta}$ DN2 cells at preleukemic and leukemic stages compared with WT DN2 cells (Figure 5B and Supplemental Figure 4). The enrichment of canonical PRC2 targets was also markedly greater in Ezh2 $2^{\Delta / \Delta}$ p5 $3^{\Delta / \Delta}$ DN1 cells than in WT DN1 cells (data not shown). However, we noted that the enrichment of PRC2 targets was slightly blunted in ETP-ALL cells than in preleukemic Ezh2 $2^{\Delta / \Delta} \mathrm{p} 53^{\Delta / \Delta}$ cells, implying that alternative mechanisms operate to repress the transcription of PRC2 targets during the leukemic transformation.

Among canonical PRC2 targets, the expression of $p 16^{\text {Ink } 4 a}$ and p19 $9^{\text {Arf }}$ was derepressed following the loss of H3K27me3 modifications at the Cdkn2a locus in Ezh2 $2^{\Delta / \Delta} \mathrm{p} 53^{\Delta / \Delta}$ cells (Figure 5C). In contrast, the expression of Nr4a3/Ncorl, a target of TCR signaling in DN2 cells (18), was significantly downregulated in Ezh2 $2^{\Delta / \Delta}$ p53 $3^{\Delta / \Delta}$ preleukemic and leukemic cells despite the loss of the H3K27me3 mark at its promoter region (Figure 5D). Correspondingly, the repression of a set of genes that are upregulated at the transition from the DN2 to DN3 stages (19) was significantly greater in ETP-ALL leukemic cells than in Ezh2 $2^{\Delta / \Delta} \mathrm{p} 53^{\Delta / \Delta}$ preleukemic cells (Figure 5E). Among these, the expression of critical $\mathrm{T}$ cell development regulators, such as Runx1, Bcl11b, and Ptcra, was specifically repressed in Ezh $2^{\Delta / \Delta} \mathrm{p} 53^{\Delta / \Delta}$ DN2 cells during the development of ETP-ALL (Figure 5F), which is consistent with the perturbed differentiation phenotype of Ezh $2^{\Delta / \Delta} \mathrm{p} 53^{\Delta / \Delta}$ leukemic cells (Figure $3, \mathrm{D}$ and $\mathrm{F}$ ).

Since Runx 1 and Bcl11b are required for $\mathrm{T}$ cell commitment and the loss of myeloid potential at the DN2b stage (20, 21), we transduced $\mathrm{CD} 44^{+} \mathrm{CD} 25^{- \text {mid }} \mathrm{DN} 1 / 2$ leukemic cells with either RUNX1 or $B c l 11 b$ and analyzed their capacity to differentiate in vitro (Figure $5 G)$. While the expression of Bcl11b failed to induce the differentiation of leukemic cells beyond the DN3 stage (data not shown), exogenous RUNX1 significantly promoted the differentiation of leukemic cells to the DN4 stage (Figure 5, H and I). Since RUNX1 loss-of-function mutations are often found in ETP-ALL patients and are associated with poor clinical outcomes $(2,22)$, Runx 1 appeared to be one of the tumor suppressor genes inactivated in this context.

The aberrant expression of some HSC and myeloid markers is a hallmark of human ETP-ALL $(1,2)$. Therefore, we analyzed the expression profiles of Ezh2 $2^{\Delta / \Delta} \mathrm{p} 53^{\Delta / \Delta}$ ETP-ALL cells by utilizing the gene sets generated from defined murine hematopoietic fractions 
A $\square \begin{aligned} & \mathrm{DN} \\ & \mathrm{DP} \\ & \mathrm{CD} 4^{+} \mathrm{SP} \\ & \mathrm{CD} 8^{+} \mathrm{SP}\end{aligned}$
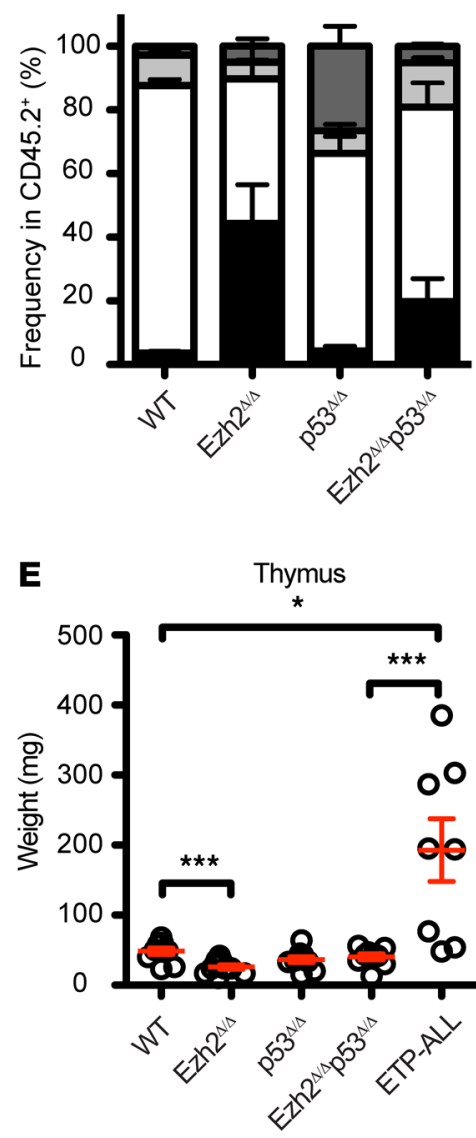

$\mathbf{F}$

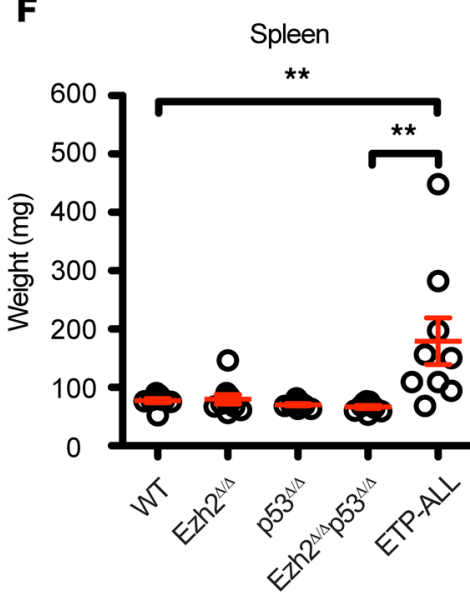

B

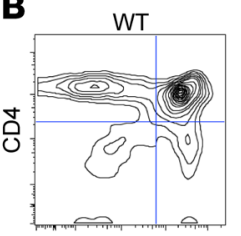

CD8

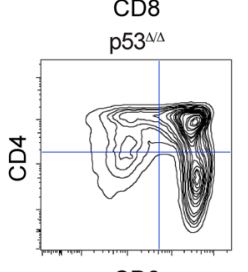

CD8

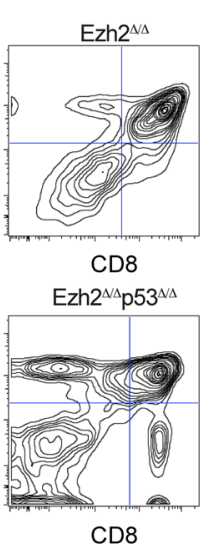

C ${ }_{\mathrm{DN} 2}^{\mathrm{DN} 1}$ 口 ${ }_{\mathrm{DN}}^{\mathrm{DN} 3}$

D $\quad W T$

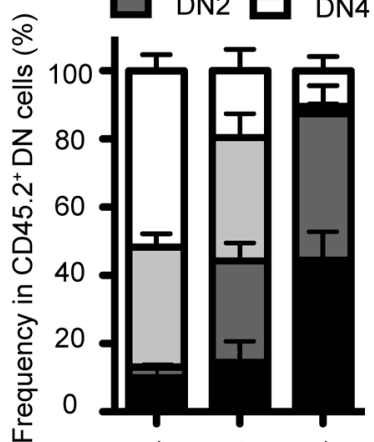

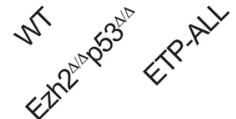

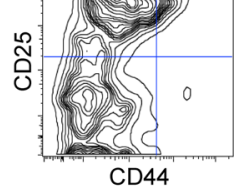

CD44

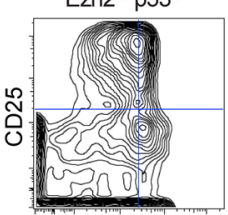

CD44

G Mouse no. 4
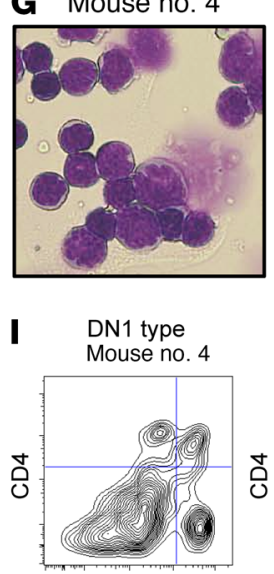

CD8

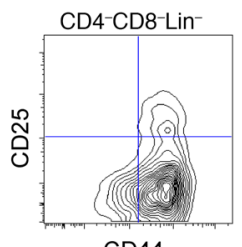

CD44

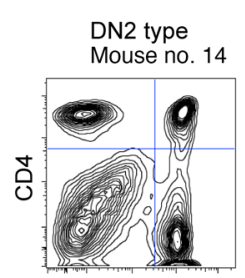

CD8

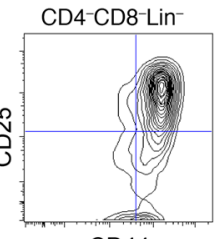

CD44
Mouse no. 6

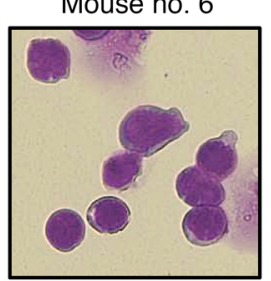

H

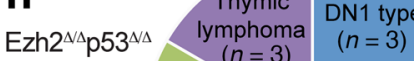

ETP-ALL subtypes

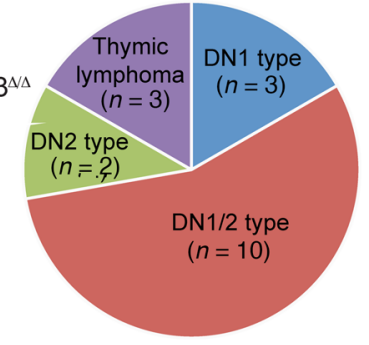

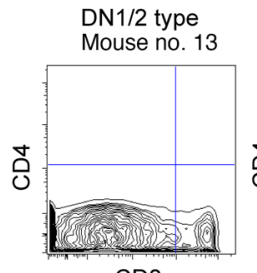

CD8

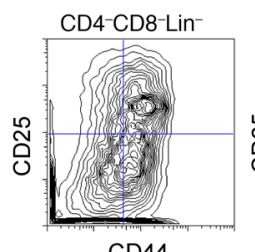

CD44

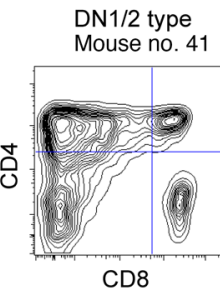

CD4-CD8-Lin ${ }^{-}$

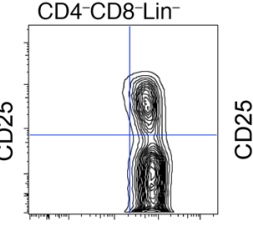

CD44

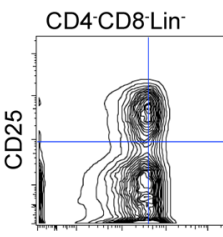

c-Kit

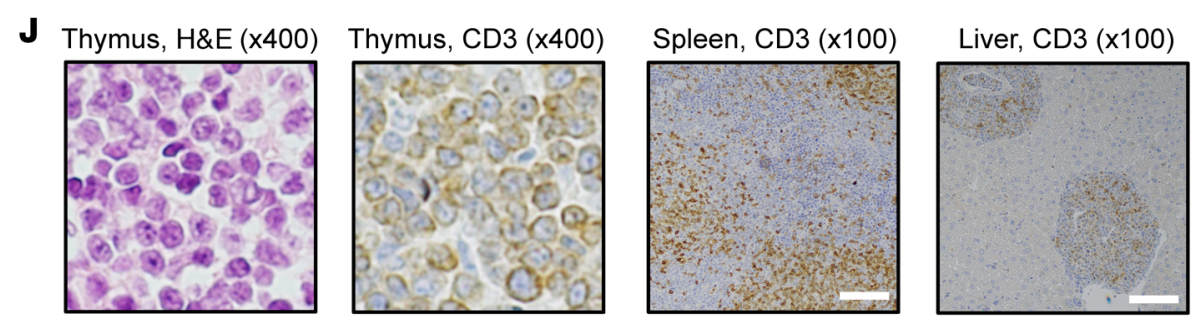

K D $\quad$ 1-J $\beta 1$ rearrangement

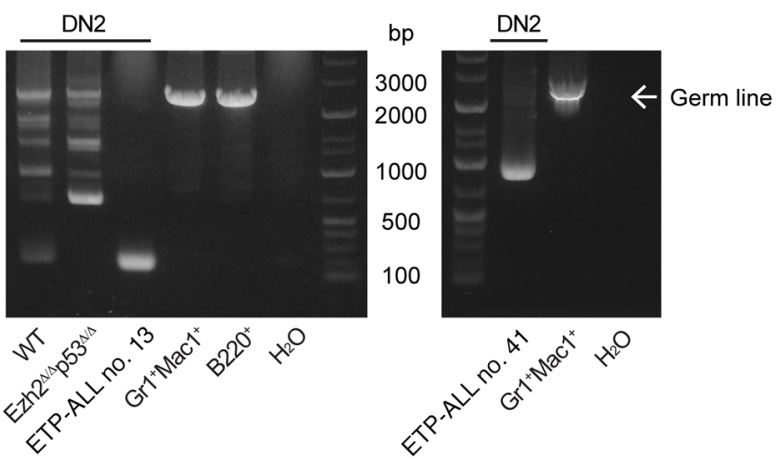


Figure 2. Ezh2 loss promoted the development of ETP-ALL in the absence of p53. (A) Proportions of CD4-CD8- DN cells among CD45.2+ cells in the thymus of WT, Ezh2 $2^{\Delta / \Delta}, \mathrm{p} 53^{\Delta / \Delta}$, and Ezh2 $2^{\Delta / \Delta} \mathrm{p} 53^{\Delta / \Delta}(n=4-7)$ mice 3 months after transplantation. Data are shown as mean \pm SEM. (B) Representative flow cytometric profiles of $\mathrm{CD} 4$ and $\mathrm{CD} 8$ expression on donor-derived CD45.2 $2^{+}$cells in the thymus of WT, Ezh2 $2^{\Delta / \Delta}, \mathrm{p} 53^{\Delta / \Delta}$, and Ezh2 $2^{\Delta / \Delta} \mathrm{p} 53^{\Delta / \Delta}$ mice. (C) Proportions of CD44+CD25- DN1, CD44+CD25+ DN2, CD44-CD25+ DN3, and CD44-CD25- DN4 cells among donor-derived CD45.2 ${ }^{+}$CD4-CD8- DN cells in the thymus $(n=4-7)$. Data are shown as mean \pm SEM. (D) Representative flow cytometric profiles of CD44 and CD25 expression on CD45.2+ DN cells in the thymus of WT and Ezh2 $2^{\Delta / \Delta} \mathrm{p} 53^{\Delta / \Delta}$ mice. (E) Thymus weight of WT $(n=10), \operatorname{Ezh}^{\Delta / \Delta}(n=10), \mathrm{p}^{\Delta / \Delta}(n=10)$, and Ezh2 $2^{\Delta / \Delta} \mathrm{p} 53^{\Delta / \Delta}(n=9)$ mice 3 months after transplantation and moribund Ezh2 ${ }^{\Delta / \Delta} \mathrm{p} 53^{\Delta / \Delta}$ ETP-ALL mice $(n=8)$ at the time of sacrifice. Data are shown as mean \pm SEM. ${ }^{*} P<0.05$; ${ }^{* * *} P<0.001$, Student's $t$ test (WT vs. Ezh2 ${ }^{\Delta / \Delta}$ ) or Mann-Whitney $U$ test (WT vs. ETP-ALL and Ezh2 $2^{\Delta / \Delta} \mathrm{p} 53^{\Delta / \Delta}$ vs. ETP-ALL). (F) Spleen weight of WT $(n=10), \operatorname{Ezh}^{\Delta / \Delta}(n=10), \mathrm{p}^{\Delta / \Delta}(n=10)$, and Ezh2 $2^{\Delta / \Delta} \mathrm{p} 53^{\Delta / \Delta}(n=9)$ mice 3 months after transplantation and moribund Ezh2 $2^{\Delta / \Delta} \mathrm{p} 53^{\Delta / \Delta}$ ETP-ALL mice $(n=8)$ at the time of sacrifice. Data are shown as mean \pm SEM. ${ }^{* *} P<0.01$, Mann-Whitney $U$ test. (G) Immature leukemic cells in the thymus of Ezh2 $2^{\Delta / \Delta}$ p53 $3^{\Delta / \Delta}$ ETP-ALL mice (mouse nos. 4 and 6 ) observed by May-Grünwald Giemsa staining. Original magnification, $\times 400$. (H) Pie chart illustrating the frequencies of the disease phenotypes of Ezh2 $2^{\Delta / \Delta} \mathrm{p} 53^{\Delta / \Delta}$ mice $(n=18)$. ETP-ALL with DN1, DN2, and DN1/2 type and thymic lymphoma were identified. (I) Representative flow cytometric profiles of CD4/CD8 expression in donor-derived CD45.2+ cells, and c-Kit, CD44, and CD25 expression in CD45.2+ Lin ${ }^{-}$cells in the thymus of each subtype of ETP-ALL shown in $\mathbf{H}$. (J) Representative histology of the thymus, spleen, and liver of an Ezh2 $2^{\Delta / \Delta}$ p53 $3^{\Delta / \Delta}$ ETP-ALL mouse observed by H\&E staining and CD3 staining. Original magnification, $\times 400$ (left panels); $\times 100$ (right panels). Scale bars: $100 \mu \mathrm{m}$. (K) Rearrangement of the TCR- $\beta$ gene in DN2 cells from WT and Ezh2 $2^{\Delta / \Delta}$ p53 $3^{\Delta / \Delta}$ mice 3 months after transplantation and ETP-ALL mice (mouse nos. 13 and 41 ) assessed by D $\beta 1-J \beta 1$ amplifications by PCR. Germline bands were observed in WT Gr1+Mac1+ neutrophils and B220+ B cells.

(23). As expected, the enrichment of the gene signature of HSCs was significantly greater in Ezh2 $2^{\Delta / \Delta} \mathrm{p} 53^{\Delta / \Delta}$ ETP-ALL cells than in WT DN2 cells (Figure 5J), supporting the human ETP-ALL-like nature of Ezh2 $2^{\Delta / \Delta} \mathrm{p} 53^{\Delta / \Delta}$ leukemic cells. We confirmed that the expression of $H l f$ and Hoxa1O was significantly stronger in Ezh $2^{\Delta / \Delta} \mathrm{p} 53^{\Delta / \Delta}$ ETPALL cells by quantitative PCR (Figure $5 \mathrm{~K}$ ). The JAK/STAT and Ras signaling pathways are generally activated in human ETP-ALL cells (2), and the loss of Ezh2 has been shown to enhance Stat3 phosphor-

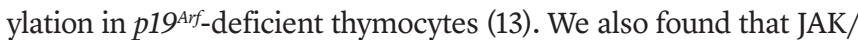
STAT and Ras signaling pathway genes were significantly activated in Ezh2 $2^{\Delta / \Delta} \mathrm{p} 53^{\Delta / \Delta}$ ETP-ALL cells (Supplemental Figure 5). The inactivation of PRC2 abrogated the transcriptional program of T cell development genes including Runx1, but resulted in the acquisition of some stem cell-associated gene signatures, leading to the enhanced proliferative capacity of leukemic cells in the absence of p53.

The loss of Ezh2 and p53 induces the propagation of DNA hypermethylation of critical $T$ cell developmental regulators during the leukemic transformation. As described above, a significant portion of the canonical Ezh2-PRC2 targets were transcriptionally silenced or downregulated despite the absence of Ezh2, suggesting that an alternative mechanism is operating in this process during leukemic transformation. We previously reported that the loss of Ezh2 induced aberrant DNA hypermethylation in a significant portion of the CGIs of PRC2 targets in the $\mathrm{Lin}^{-} \mathrm{Sca}-\mathrm{1}^{+} \mathrm{c}-\mathrm{Kit}^{+}$(LSK) hematopoietic stem/progenitor cells of Tet 2 hypomorphic mice $\left(\operatorname{Tet} 2^{K D / K D}\right)$, and found a critical role for this epigenetic switch in the pathogenesis of MDS that developed in Ezh2 $2^{\Delta / \Delta} \mathrm{Tet} 2^{\mathrm{KD} / \mathrm{KD}}$ mice (24). In order to elucidate whether this epigenetic switch also took place in Ezh2 $2^{\Delta / \Delta} \mathrm{p} 53^{\Delta / \Delta}$ ETP-ALL cells, we performed reduced representative bisulfite sequencing (RRBS) on DN2 cells isolated from WT, Ezh2 $2^{\Delta / \Delta}, \mathrm{p} 53^{\Delta / \Delta}$, and Ezh2 $2^{\Delta / \Delta} \mathrm{p} 53^{\Delta / \Delta}$ mice at the preleukemic stage and Ezh2 $2^{\Delta / \Delta} \mathrm{p} 53^{\Delta / \Delta}$ ETP-ALL DN2 cells. While neither Ezh2 $2^{\Delta / \Delta}$ cells nor $\mathrm{p} 53^{\Delta / \Delta}$ cells significantly gained hyperdifferentially methylated regions (hyper-DMRs) at the promoter regions $(-2.5 \mathrm{~kb}$ to $+0.5 \mathrm{~kb}$ from transcription start site [TSS]) compared with WT cells, DNA hypermethylation was mild in preleukemic Ezh2 $2^{\Delta / \Delta} \mathrm{p} 53^{\Delta / \Delta}$ cells and robust in Ezh $2^{\Delta / \Delta} \mathrm{p} 53^{\Delta / \Delta}$ leukemic cells (Figure 6A). Notably, Ezh2 ${ }^{\Delta / \Delta} \mathrm{p} 53^{\Delta / \Delta}$ leukemic cells established hyper-DMRs at a considerably larger portion of promoter regions than was seen in WT cells and exhibited only a few hypo-DMRs (Figure 6B). These results indicate that the deletion of $E z h 2$ and $p 53$ alone was not sufficient to induce aberrant DNA hypermethylation, but induced aberrant DNA hypermethylation in a combinatorial manner, particularly during the development of leukemia. Gene ontology (GO) enrichment analyses revealed that hyper-DMRs in Ezh2 ${ }^{\Delta / \Delta} \mathrm{p} 53^{\Delta / \Delta}$ leukemic cells were significantly enriched in genes associated with development, cell adhesion, differentiation, and transcription factor activity (Figure 6C), while hypo-DMRs were enriched in a few categories, such as lipid catabolic processes (Figure 6C), suggesting that DNA hypermethylation plays a role in the development of ETP-ALL.

We then attempted to elucidate the relationship of epigenetic modifications between DNA methylation and H3K27me3 levels in Ezh2 $2^{\Delta / \Delta} \mathrm{p} 53^{\Delta / \Delta}$ leukemic cells at the promoter regions and found that hyper-DMRs were strongly associated with lower levels of H3K27me3 in Ezh2 ${ }^{\Delta / \Delta} \mathrm{p} 53^{\Delta / \Delta}$ leukemic cells than in WT cells (Figure 6D and Supplemental Figure 6). Furthermore, a significant portion $(38 \%)$ of hyper-DMRs in Ezh2 $2^{\Delta / \Delta} \mathrm{p} 53^{\Delta / \Delta}$ leukemic cells were observed in the canonical PRC2 targets defined in WT DN1 cells (Supplemental Figure 7). Indeed, the PRC2 targets in WT DN1 cells acquired significantly higher levels of DNA methylation than all RefSeq genes in Ezh2 $2^{\Delta / \Delta} \mathrm{p} 53^{\Delta / \Delta}$ leukemic cells (Figure 6E). In contrast, the PRC2 targets did not gain DNA hypermethylation in Ezh2 ${ }^{\Delta / \Delta}$ cells, compared with WT cells (Figure 6E). These results indicate that the loss of both Ezh2 and p53 induce the epigenetic switch from H3K27me3 to DNA hypermethylation at the PRC2 target promoter regions during the development of leukemia.

DNA methylation levels at promoter regions do not necessarily correlate with gene expression levels $(25,26)$. Consistent with this finding, there was no correlation between the levels of DNA methylation and gene expression in Ezh2 ${ }^{\Delta / \Delta}, \mathrm{p} 53^{\Delta / \Delta}$, and Ezh2 $2^{\Delta / \Delta}$ p5 $3^{\Delta / \Delta}$ DN2 cells and Ezh2 ${ }^{\Delta / \Delta} \mathrm{p} 53^{\Delta / \Delta}$ leukemic cells in the present study (Figure 6F and Supplemental Figure 8). However, we found an epigenetic switch from H3K27me3 to DNA methylation at the promoters of genes encoding nonhematopoietic developmental regulators (e.g., Tbx1 and Sox9) as well as T cell development regulators (e.g., Runx1 and Nr4a3) (Figure 6G) associated with reduced levels of expression in Ezh2 $2^{\Delta / \Delta} \mathrm{p} 53^{\Delta / \Delta}$ leukemic cells (Figure 5, D and F). These results imply that the epigenetic switch reinforced the transcriptional repression of key $\mathrm{T}$ cell regulator genes, thereby promoting the pathogenesis of ETP-ALL.

Aberrant DNA hypermethylation contributes to the pathogenesis of ETP-ALL. In order to understand the molecular 
A

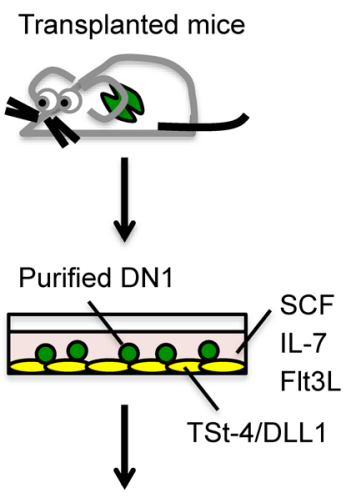

7-14 days
B

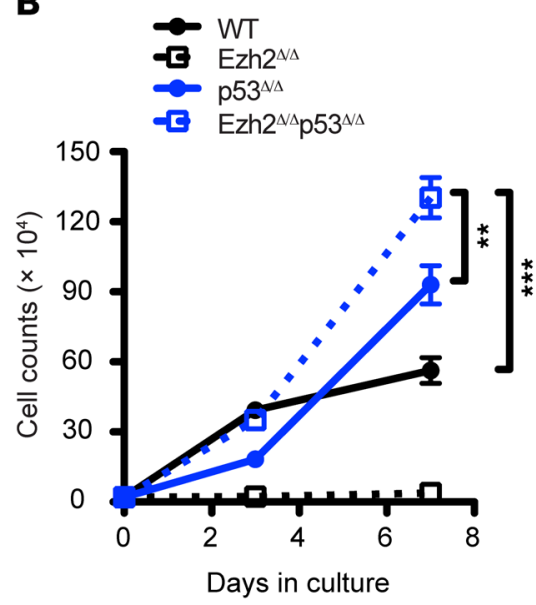

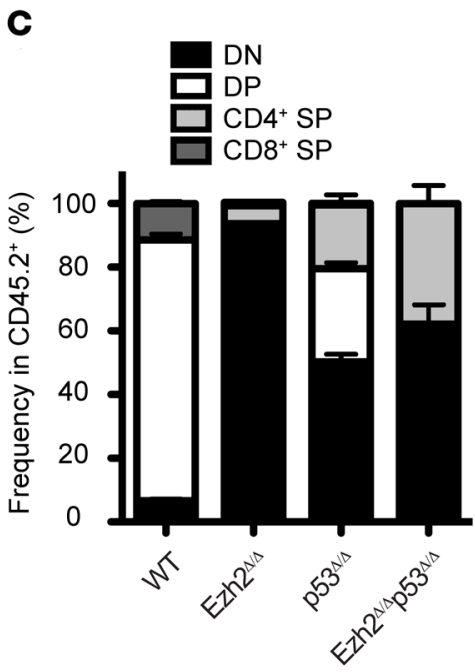

D

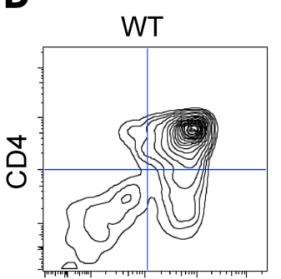

CD8

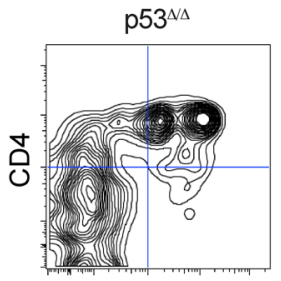

CD8

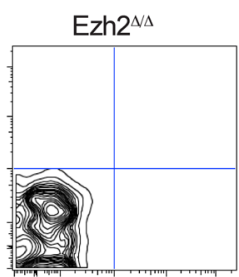

CD8

Ezh2 ${ }^{N / \mathrm{p}} 53^{\mathrm{NA}}$

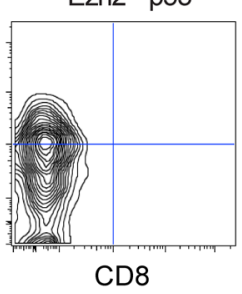

E

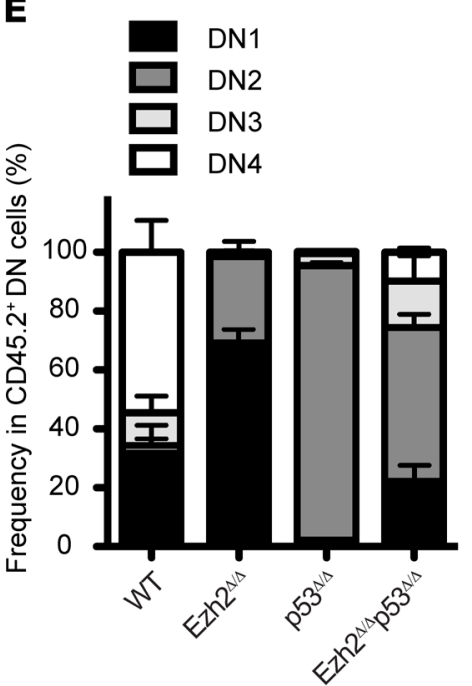

$\mathbf{F}$
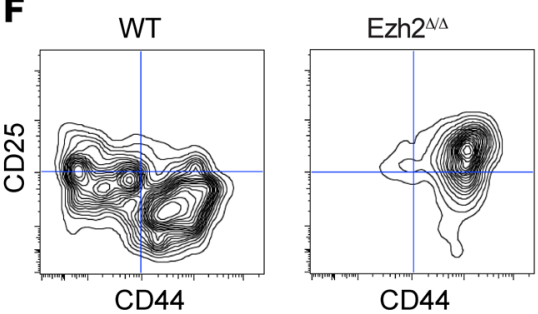

p53 $3^{\Delta \Delta}$

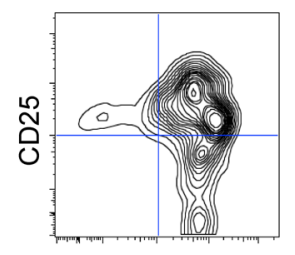

CD44
$\mathrm{Ezh} 2^{\mathrm{N} / \Delta} \mathrm{p} 53^{\mathrm{N} / \Delta}$

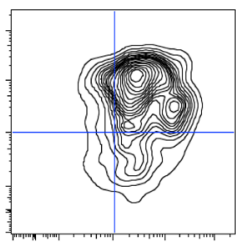

CD44

Figure 3. The loss of Ezh2 impeded T cell differentiation at the CD4-CD8- stage. (A) Experimental schematic of the procedure to examine the T cell differentiation potential of purified CD45.2+CD44+CD25-Lin- DN1 cells. DN1 cells were cultured with TSt-4/DLL1 stromal cells in the presence of SCF, IL-7, and Flt3L ( $10 \mathrm{ng} / \mathrm{ml}$ ) for 7 to 14 days. (B) Total cell counts of WT (black line), Ezh2 $2^{\Delta / \Delta}$ (black broken line), $\mathrm{p} 53^{\Delta / \Delta}$ (blue line), and Ezh2 $2^{\Delta / \Delta} \mathrm{p} 53^{\Delta / \Delta}$ (broken blue line) cells during culture $(n=3-6)$. Data are shown as mean \pm SEM. ${ }^{* *} P<0.01$; ${ }^{* * *} P<0.001$, Student's $t$ test. (C) Proportions of CD4 ${ }^{+}$CD8 ${ }^{+}$cells in CD45.2 ${ }^{+}$cells on day 7 of WT, Ezh2 $2^{\Delta / \Delta}, p 53^{\Delta / \Delta}$, and Ezh2 $2^{\Delta / \Delta} \mathrm{p} 53^{\Delta / \Delta} \mathrm{DN1}$ cell culture $(n=3-6)$. Data are shown as mean \pm SEM. (D) Representative flow cytometric profiles of CD4 and CD8 expression in WT, Ezh2 $2^{\Delta / \Delta}$, p53 $3^{\Delta / \Delta}$, and Ezh2 ${ }^{\Delta / \Delta} \mathrm{p} 53^{\Delta / \Delta} \mathrm{CD} 45.2^{+}$cells on day 7 of the culture. (E) Proportions of DN1, DN2, DN3, and DN4 cells in CD45.2+CD4-CD8- DN cells on day 7 of the culture $(n=3-6)$. Data are shown as mean \pm SEM. (F) Representative flow cytometric profiles of CD44 and CD25 expression in CD45.2+CD4-CD8- cells on day 7 of the culture. Data are representative of 2 independent experiments.

mechanisms underlying the epigenetic switch, we examined expression of Dnmt1, Dnmt3a, and Dnmt3b in DN2 cells by quantitative PCR and found that Ezh2 $2^{\Delta / \Delta} \mathrm{p} 53^{\Delta / \Delta}$ leukemic cells expressed Dnmt genes at levels similar to those of DN2 cells with other genotypes and Ezh $2^{\Delta / \Delta} \mathrm{p} 53^{\Delta / \Delta}$ cells at the preleukemic stage (Supplemental Figure 9). We next asked whether pathogenic DNA hypermethylation in these key genes contributed to leukemic transformation. We first knocked down Dnmt1, Dnmt3a, or Dnmt3b by utilizing individual shRNA vectors (Supplemental Figure 10) and assessed the impact of knocking down these methyltransferases in EPT-ALL DN1/2 cells. We found that knockdown of individual Dnmt genes was not effective in canceling the differentiation block of ETP-ALL cells and only partially promoted the differentiation of ETP-ALL cells beyond the DN3 stage, but hardly into DP cells (Supplemental Figure 11), implying that Dnmt1, Dnmt3a, and Dnmt3b establish aberrant DNA hypermethylation and induce the differentiation block in a combinatorial manner.

We then assessed the efficacy of the DNA hypomethylating agent DAC at canceling the differentiation block of Ezh $2^{\Delta / \Delta} \mathrm{p} 53^{\Delta / \Delta}$ leukemic cells. Under in vitro differentiation conditions, the DAC treatment did not affect the growth or differentiation of WT DN1/2 cells (data not shown). In contrast, DAC markedly suppressed the growth of Ezh2 $2^{\Delta / \Delta} \mathrm{p} 53^{\Delta / \Delta} \mathrm{CD} 44^{+} \mathrm{CD} 25^{\text {mid }}$ leukemic cells in a dosedependent manner (Figure 7A) and successfully induced the differentiation of leukemic cells into the DP and $\mathrm{CD} 8^{+} \mathrm{SP}$ stages (Figure 7, B and C). The recovery of the expression of Nr4a3 and Bcl11b was significantly greater, while the expression of Runx 1 was moderately 
A

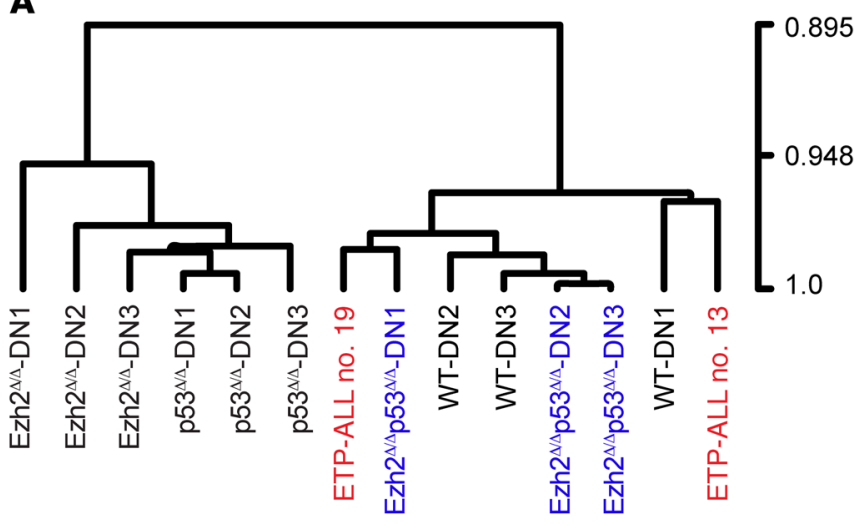

C Up-regulated genes

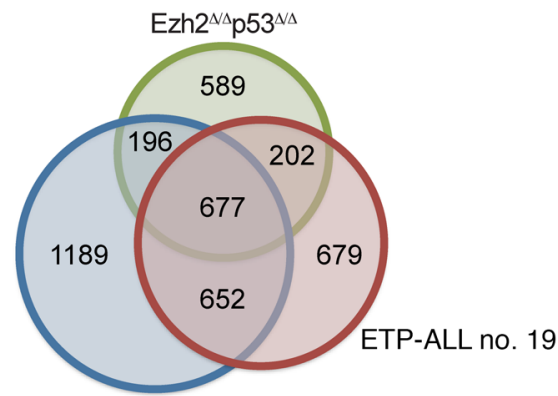

ETP-ALL no. 13
B

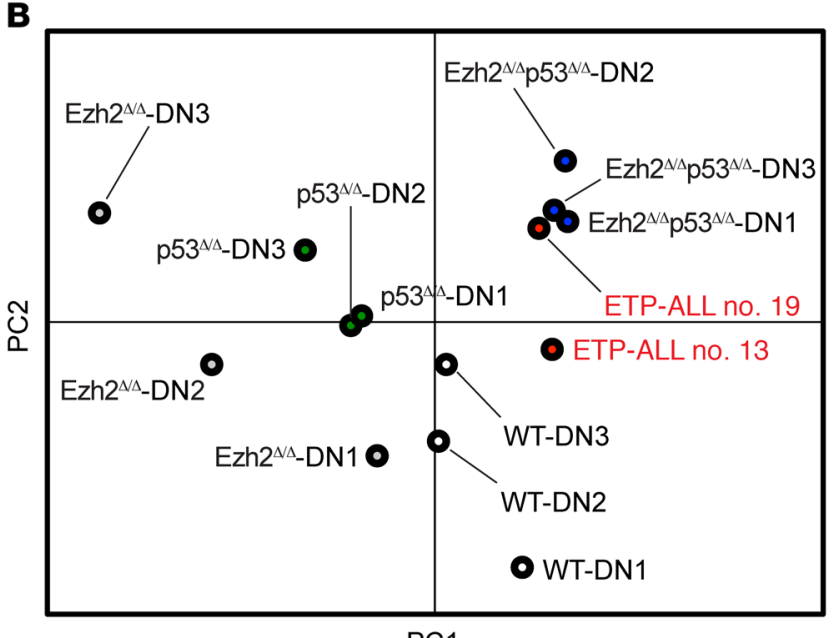

PC1

Figure 4. Concurrent loss of Ezh2 and p53 impaired the transcriptional program of T cell differentiation. (A) Hierarchical clustering based on total gene expression in DN1, DN2, and DN3 cells isolated from WT, Ezh2 $2^{\Delta / \Delta}, \mathrm{p} 53^{\Delta / \Delta}$, and Ezh2 $2^{\Delta / \Delta} \mathrm{p} 53^{\Delta / \Delta}$ mice 3 months after transplantation ( $\left.n=5-10\right)$ and in DN2 leukemic cells isolated from 2 distinct Ezh2 $2^{\Delta / \Delta} \mathrm{p} 53^{\Delta / \Delta}$ ETP-ALL mice (mouse nos. 13 and 19). Linkage scores are indicated on the right. (B) A principal component (PC) analysis based on total gene expression in DN1, DN2, and DN3 cells isolated from WT mice (white circles), Ezh2 ${ }^{\Delta / \Delta}$ mice (gray circles), $\mathrm{p} 53^{\Delta / \Delta}$ mice (green circles), Ezh2 $2^{\Delta / \Delta} \mathrm{p} 53^{\Delta / \Delta}$ mice at a predisease stage (blue circles), and DN2 leukemic cells from Ezh2 $2^{\Delta / \Delta} \mathrm{p} 53^{\Delta / \Delta}$ ETP-ALL mice (red circles). (C) Venn diagrams showing overlaps of upregulated and downregulated genes (left and right panels, respectively) between DN2 cells from Ezh2 $2^{\Delta / \Delta} \mathrm{p} 53^{\Delta / \Delta}$ mice 3 months after transplantation and Ezh2 ${ }^{\Delta / \Delta} \mathrm{p} 53^{\Delta / \Delta}$ ETP-ALL mice (nos. 13 and 19), relative to gene expression in WT DN2 cells.

stronger in DAC-treated leukemic cells than in dimethylsulfoxidetreated (DMSO-treated) leukemic cells (Figure 7D), indicating that the restored expression of $\mathrm{T}$ cell development regulators coordinated to enforce the terminal differentiation of ETP leukemic cells.

In order to examine the impact of DAC on pathogenic DNA hypermethylation in Ezh2 $2^{\Delta / \Delta} \mathrm{p} 53^{\Delta / \Delta}$ leukemic cells, we performed RRBS on DMSO-treated DN2 leukemic cells and DAC-induced DN4 cells in the culture. We found that DNA methylation levels at CGIs and promoter regions were significantly lower in DACinduced DN4 cells than in control DN2 cells (Figure 7E), as represented at the $\mathrm{Nr} 4 \mathrm{a} 3$ locus (Figure 7F). In addition, GO analysis revealed that hypo-DMRs in DAC-induced DN4 cells compared with control DN2 cells were significantly enriched in genes with development, cell adhesion, differentiation, and transcription factor activity (Figure $7 \mathrm{G}$ ). These results indicate that pathogenic DNA hypermethylation in key regulatory genes abrogated the operation of the normal network of $\mathrm{T}$ cell development genes and contributed to the pathogenesis of ETP-ALL.

Finally, in order to examine whether DAC inhibits the development of ETP-ALL in vivo, we transplanted Ezh2 $2^{\Delta / \Delta}$ p53 $3^{\Delta / \Delta}$ ETP-ALL cells $\left(1 \times 10^{6}\right.$ cells $)$ together with $2 \times 10^{6} \mathrm{WT}$ $\mathrm{BM}$ cells into lethally irradiated recipient mice and treated the recipient mice with DAC $(0.2 \mathrm{mg} / \mathrm{kg})$ or DMSO from 3 weeks after transplantation. Although DAC did not release the differentiation block of leukemic cells in this setting (data not shown), we observed that DAC impeded the expansion of leukemic cells, as evident in the reduced proportion of leukemic cells in the PB (Figure 7H). Consequently, DAC treatment extended the survival of recipient mice (Figure 7I), although the statistical significance was marginal, supporting the idea that DAC would be warranted for a therapy on patients with ETP-ALL harboring the EZH2 mutation.

\section{Discussion}

ETP-ALL is a new disease entity of T cell malignancies with a poor clinical outcome. ETP-ALL cells harbor high frequencies of deletions and/or loss-of-function mutations in PRC2, including $E Z H 2$. $E Z H 2$ has been characterized as an oncogene in many cancers, including B cell lymphoma, in which gain-of-function mutations in EZH2 play a pathogenic role $(27,28)$, and acute myeloid leukemia (AML) $(29,30)$. Recently, gain-of-function mutations in EZH2 have been shown to be neomorphic because Ezh2 ${ }^{\mathrm{Y} 641 \mathrm{~F}}$ globally increased the levels of H3K27me3, but also induced loss of H3K27me3 modification in a region-dependent manner that 
A

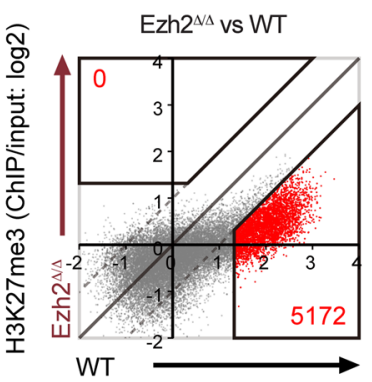

H3K27me3 (ChIP/input: $\log _{2}$ )

C

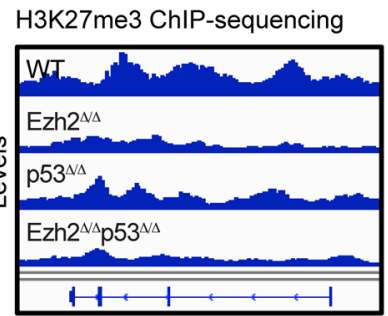

Cdkn2a (p16 $6^{\left.\text {nk4a } / p 19^{A R F}\right)}$

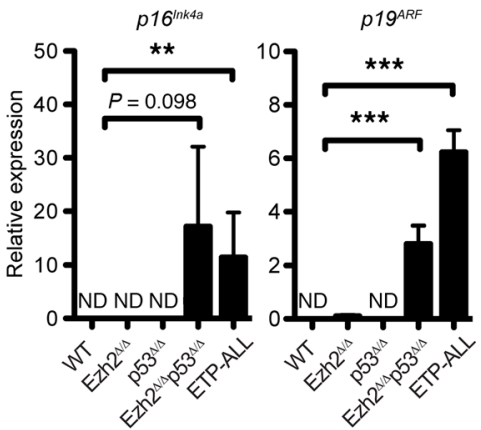

B

Canonical PRC2 target genes

Ezh2 ${ }^{\Delta / \Delta} p 53^{\Delta / \Delta}$ vs WT

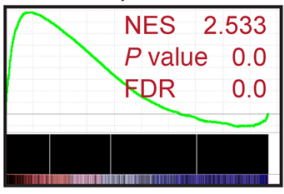

ETP no. 13 vs. WT
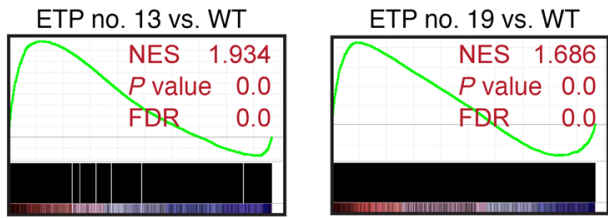

E

Up-regulated genes in the transition from DN2 to DN3 ETP no. 13 vs. pre-disease ETP no. 19 vs. pre-disease

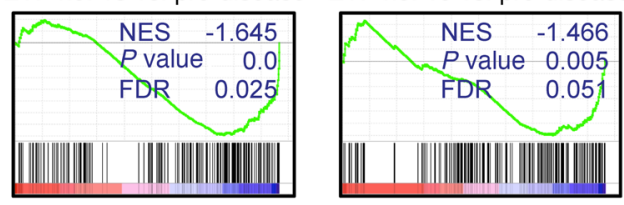

$\mathbf{F}$

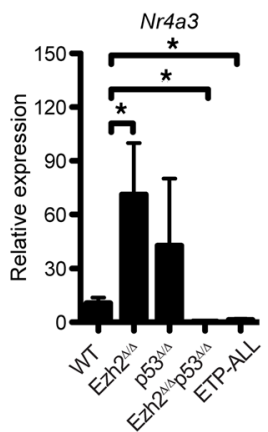

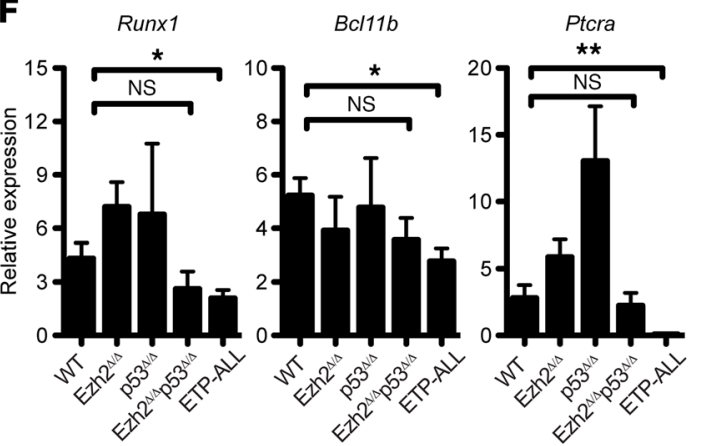

G

$\underset{\text { Reduction of IL-7 }}{\downarrow}$

9 days culture

J

HSCs signature

ETP no. 13 vs. WT

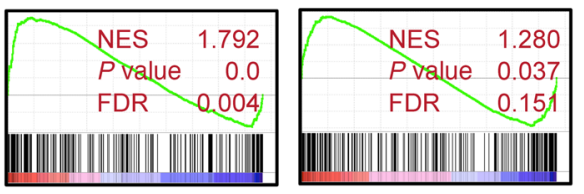

H CD4-CD8-Lin- gated

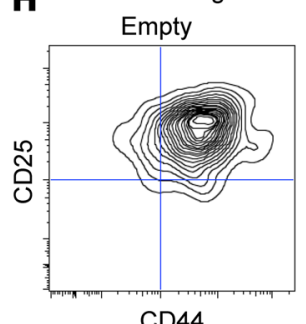

CD44

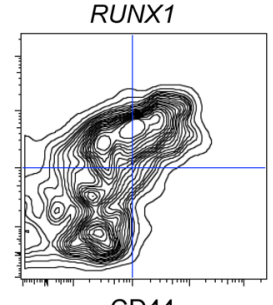

CD44
I

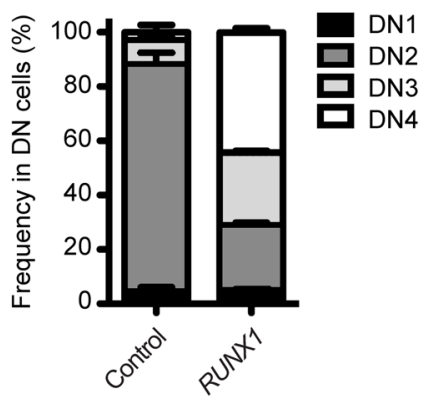

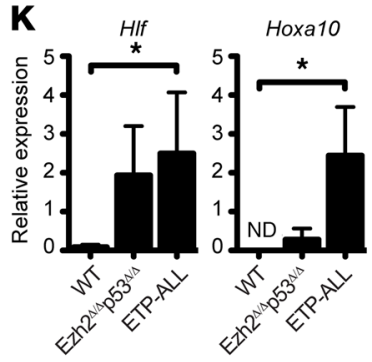


Figure 5. Ezh2 loss led to the silencing of critical T cell lineage determinants. (A) Scatter plots showing the relationship of the fold enrichment values (ChIP/input) of H3K27me3 (TSS $\pm 2.0 \mathrm{~kb}$ of RefSeq genes) between WT, Ezh2 ${ }^{\Delta / \Delta}, \mathrm{p} 53^{\Delta / \Delta}$, and Ezh2 $2^{\Delta / \Delta} \mathrm{p} 53^{\Delta / \Delta}$ DN1 (CD44 ${ }^{+}$CD25-Lin') cells 2 months after the deletion of Ezh2 and/or p53. (B) GSEA plots for canonical PRC2 target genes comparing DN2 cells from WT mice to DN2 cells from Ezh2 ${ }^{\Delta / \Delta}$ $\mathrm{p} 53^{\Delta / \Delta}$ mice at a predisease stage and 2 distinct Ezh2 $2^{\Delta / \Delta} \mathrm{p} 53^{\Delta / \Delta}$ ETP-ALL mice (nos. 13 and 19). Normalized enrichment score (NES), nominal $P$ value, and FDR $q$ values are indicated. (C) ChIP-seq view of H3K27me3 levels at the Cdkn2a locus in WT, Ezh2 $2^{\Delta / \Delta}, \mathrm{p} 53^{\Delta / \Delta}$, and Ezh2 $2^{\Delta / \Delta} \mathrm{p} 53^{\Delta / \Delta}$ DN1 cells (top), and a quantitative RT-PCR analysis of the expression of $p 16^{\operatorname{lnk} 4 a}$ and $p 19^{\text {arf in }}$ DN2 cells isolated from WT $(n=12)$, Ezh2 $2^{\Delta / \Delta}(n=6), p 53^{\Delta / \Delta}(n=6)$, and Ezh2 $2^{\Delta / \Delta}$ $\mathrm{p} 53^{\Delta / \Delta}(n=8)$ mice 3 months after transplantation and ETP-ALL mice $(n$ = 8) (bottom). (D) ChIP-seq view of H3K27me3 levels at the Nr4a3 locus in WT, Ezh2 ${ }^{\Delta / \Delta}, \mathrm{p} 53^{\Delta / \Delta}$, and Ezh2 $2^{\Delta / \Delta} \mathrm{p} 53^{\Delta / \Delta}$ DN1 cells (top), and quantitative RT-PCR data of the expression of Nr4a3 in DN2 cells isolated from WT ( $n=$ 12), Ezh2 $2^{\Delta / \Delta}(n=6), \mathrm{p} 53^{\Delta / \Delta}(n=6)$, and Ezh2 $2^{\Delta / \Delta} \mathrm{p} 53^{\Delta / \Delta}(n=8)$ mice 3 months after transplantation and ETP-ALL mice $(n=8)$ (bottom) ${ }^{*} P<0.001$. (E) GSEA plots for $\mathrm{T}$ cell differentiation regulators, which were upregulated at the transition from DN2 to DN3 stages (19), comparing the DN2 cells of 2 distinct Ezh2 $2^{\Delta / \Delta} \mathrm{p}^{5} 3^{\Delta / \Delta}$ ETP-ALL mice (nos. 13 and 19) with those of Ezh2 ${ }^{\Delta /}$ ${ }^{\Delta} \mathrm{p} 53^{\Delta / \Delta}$ mice 3 months after transplantation. (F) Quantitative RT-PCR analysis of the expression of Runx1, Bcl11b, and Ptcra in DN2 cells isolated from WT ( $n=12)$, Ezh2 $^{\Delta / \Delta}(n=6), p 53^{\Delta / \Delta}(n=6)$, and Ezh2 $2^{\Delta / \Delta} \mathrm{p}^{5} 3^{\Delta / \Delta}(n=8)$ mice 3 months after transplantation and Ezh2 $2^{\Delta / \Delta} \mathrm{p} 53^{\Delta / \Delta}$ ETP-ALL mice $(n=8)$. (C) Experimental schematic of the procedure to examine the T cell differentiation of Ezh2 ${ }^{\Delta / \Delta} \mathrm{p} 53^{\Delta / \Delta}$ leukemic cells. (H) Representative flow cytometric profiles of CD44 and CD25 expression in CD4-CD8- Lin-CFP+ transduced leukemic cells on day 9 of the culture $(n=3)$. Data are representative of 2 independent experiments. (I) Proportions of DN1, DN2, DN3, and DN4 cells in CD4-CD8- Lin-GFP+ transduced leukemic cells on day 9 of the control and RUNX1 cultures $(n=3)$. (J) GSEA plots for the gene expression signatures of HSCs comparing DN2 cells isolated from WT mice and 2 distinct Ezh2 ${ }^{\Delta /}$ ${ }_{\mathrm{p}} 53^{\Delta / \Delta}$ ETP-ALL mice. (K) Quantitative RT-PCR analysis of the expression of HIf and Hoxa10 in DN2 cells isolated from WT $(n=12)$ and Ezh2 $2^{\Delta / \Delta} \mathrm{p}^{\Delta / \Delta}$ $(n=8)$ mice 3 months after transplantation and Ezh2 $2^{\Delta / \Delta} \mathrm{p} 53^{\Delta / \Delta} \mathrm{ETP}-\mathrm{ALL}$ mice $(n=8)$. (C, $\mathbf{D}, \mathbf{F}$, and $\mathbf{K})$ Data are shown as mean \pm SEM. ${ }^{*} P<0.05$; ${ }^{* *} P<0.01 ;{ }^{* *} P<0.001$, Student's $t$ test (F $\left.[R u n \times 1, B c / 11 b]\right)$; Mann-Whitney $U$ test (C, D, K, $\mathbf{F}[$ Ptcra $]$ ).

was associated with increased transcription of target genes (31). In contrast, EZH2-PRC2 functions as a tumor suppressor in MDS as well as $\mathrm{T}$ cell malignancies. We previously demonstrated how the hematopoietic cell-specific deletion of Ezh2 promoted the development of MDS and/or MPN diseases in concert with the loss of Tet2, RUNX1 mutants, or the JAK2 $2^{\mathrm{V} 671 F}$ mutant (8-10). We and others also previously reported that Ezh2-deficient mice showed impaired $\mathrm{T}$ cell production due to perturbed differentiation at the DN cell stage $(3,32)$. We subsequently reported that the serial transplantation of Ezh2-deficient BM cells induced CD8 ${ }^{+}$T-ALL, but not ETP-ALL, despite its low frequency (11). In the present study, we demonstrated that the loss of Ezh2 and $p 53$ collaborated

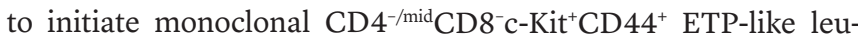
kemia in mice, which recapitulated ETP-ALL in patients. These results indicate that Ezh2 functions to ensure T cell development at multiple differentiation stages from BM HSCs to mature T cells and that its inactivation promotes ETP and non-ETP T-ALL in a context-dependent manner.

Early $\mathrm{T}$ cell development depends on the proliferation and differentiation of multipotent progenitor cells that undergo $\mathrm{T}$ cell commitment and TCR rearrangement at the CD4-CD8- DN stage. These processes are initiated by Notch signaling in the thymus and are sequentially operated by networks of differentia- tion regulator genes. To this end, transcriptional and epigenetic machineries function to promote $\mathrm{T}$ cell commitment and silence the expression of stem and progenitor cell genes, resulting in the loss of other lineage potentials, including myeloid cells (33). Transcription factor networks, including $B c l 11 b$, are required for $\mathrm{T}$ cell commitment and the loss of myeloid cell potential at the DN2b stage $(20,21)$. In the present study, we demonstrated that Ezh2p53-deficient leukemic cells suppressed the expression of pivotal genes for T cell development, such as Runx1 and Bcl11b, but sustained the expression of stem cell signature genes, which are associated with the self-renewal capacity of normal c-Kit ${ }^{+}$ETP cells. In the $N R a s^{\mathrm{Q} 61 K} C d k n 2 a^{-/-}$ETP-ALL mouse model previously reported, stem and myeloid progenitor signature genes, including Hoxa9, were aberrantly expressed in Ezh2-deficient leukemic cells because Ezh2-PRC2 directly suppresses Hoxa9 in this setting. The forced expression of Hoxa9 promoted the proliferation of NRas ${ }^{Q 61 K} C d k n 2 a^{-/-}$ETP-ALL cells (13) and also the transformation of Ezh2-deficient MDS to AML (9). However, Ezh2p53deficient ETP leukemic cells did not show the increased expression of Hoxa9 or reduced levels of H3K27me3 at the promoter region of Hoxa9 (data not shown). This discrepancy may be due to the different experimental strategies used to induce ETPALL-like cells in vitro versus in vivo. Nevertheless, the expression of stem and myeloid progenitor signature genes was enhanced in both ETP-ALL mouse models. Further studies are needed in order to elucidate how the inappropriate expression of these genes promotes the transformation of ETPs.

We recently showed that an epigenetic switch from $\mathrm{H} 3 \mathrm{~K} 27 \mathrm{me} 3$ to DNA methylation occurs following the loss of Ezh2 in Tet $2^{\mathrm{KD} / \mathrm{KD}}$ mice and maintains the transcriptional repression of a significant portion of PRC2 targets, thereby contributing to the progression of MDS (24). We found that this epigenetic switch was accentuated at the promoter regions of pivotal transcriptional regulator genes in Ezh2p53-deficient ETP-ALL cells, the silencing of which impedes the proper ensemble of transcription factors for T cell development. Correspondingly, neither RUNX1 nor Bcl11b transduction alone induced the differentiation of ETP-ALL cells into mature $\mathrm{CD} 4^{+} \mathrm{CD} 8^{+}$cells in vitro; however, DAC treatment resulted in the terminal differentiation of ETP-ALL cells following the reactivation of a set of genes encoding transcriptional regulators of $\mathrm{T}$ cell development. These results indicate that the DNA methylation-mediated inhibition of the gene networks of $\mathrm{T}$ cell development, rather than that of a single transcription factor, was largely responsible for the differentiation block of ETPs and aberrant expression of stem/myeloid genes during the development of ETP-ALL. A functional link between DNA methylation and PRC2 has been implicated in the regulation of the biological processes of development, differentiation, and cancer $(34,35)$. Previous studies showed that DNA hypermethylation in cancer, including leukemia, correlated with bivalent genes marked by trithorax groupmediated H3K4me3 and PRC2-mediated H3K27me3, which are activated upon the differentiation of ES cells $(36,37)$. As we previously reported in Ezh2-deficient MDS cells (24), Ezh2p53-deficient ETP-ALL cells acquired higher levels of DNA methylation at the CGI promoter regions of PRC2 target genes. Of interest, Fbxl10/ $\mathrm{Kdm} 2 \mathrm{~b}$, a component of noncanonical PRC1, has been shown to protect the CGI promoters of polycomb-bound genes from DNA 
A

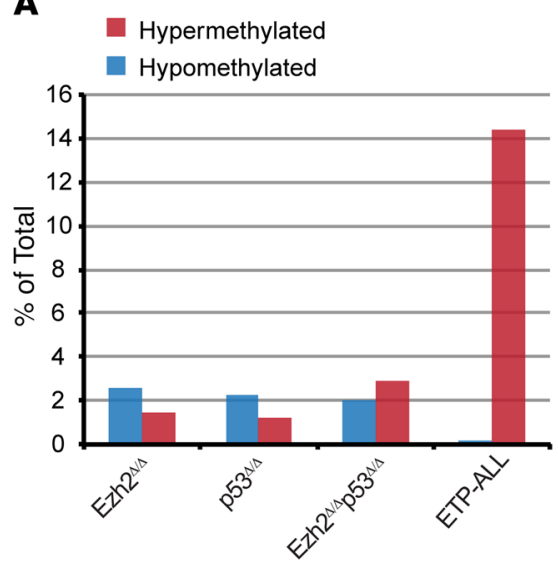

D

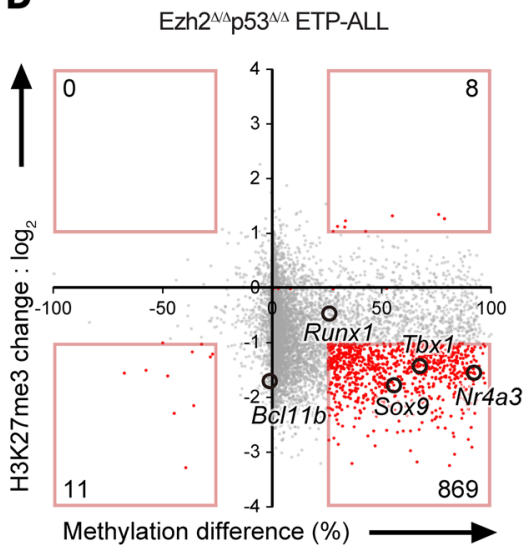

F

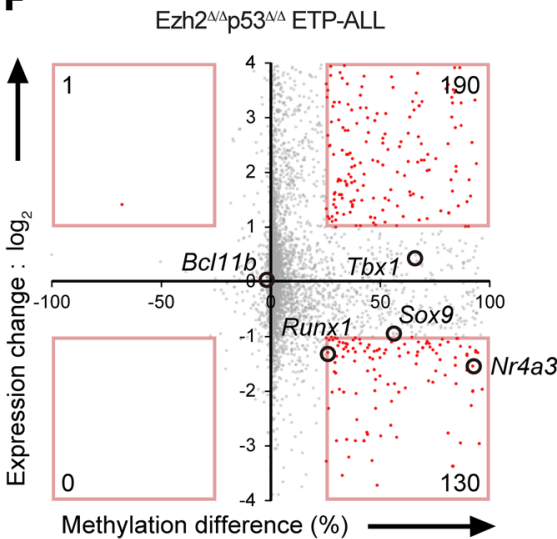

B

Ezh2 $2^{\wedge \wedge}$ p53 $3^{\Delta \wedge}$ ETP-ALL versus WT

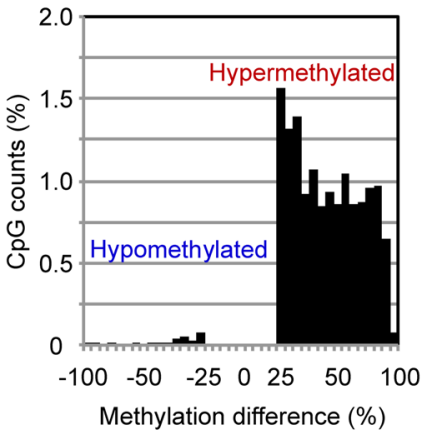

C

\begin{tabular}{llllllll} 
Hypermethylated & \multicolumn{1}{c}{$P$ value $\left(\log _{10}\right)$} \\
Hypomethylated & 0 & -2 & -4 & -6 & -8 & -10 & -12
\end{tabular} Cell adhesion

Multicellular organisma development

Angiogenesis

Cell differentiation

Sequence-specific DNA binding transcription factor activity

Elevation of cytosolic calcium ion concentration

Lipid catabolic process

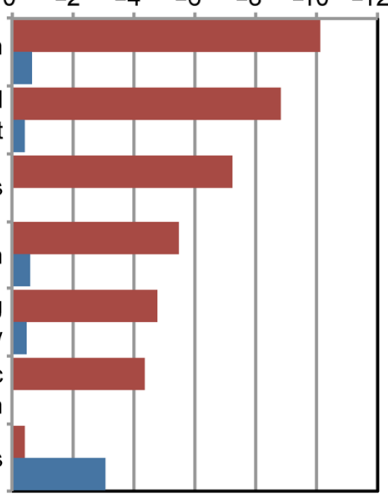

E

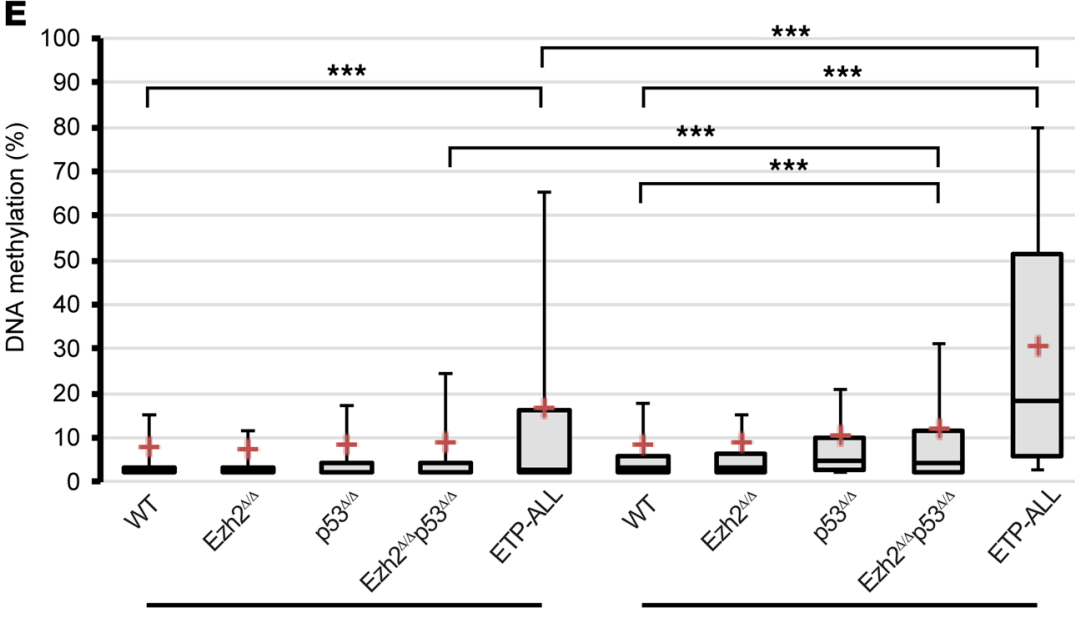

All genes

PRC2 targets

G
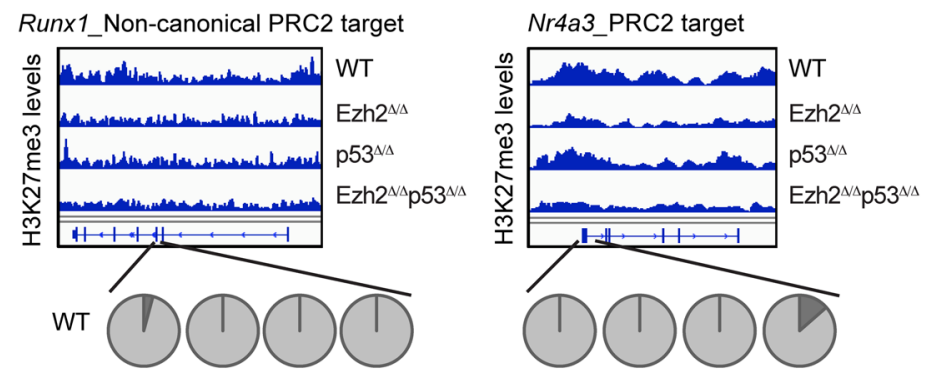

Avg. methylation $=0.69 \%$

Ezh2 $^{\Delta / \Delta}$

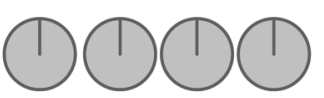

Avg. methylation $=3.1 \%$

p53 $3^{\Delta / \Delta}$

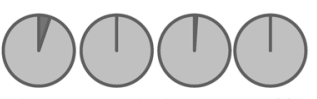

Avg. methylation $=0.45 \%$

Ezh2 ${ }^{\Delta / \Delta} \mathrm{p} 53^{\Delta / \Delta}$

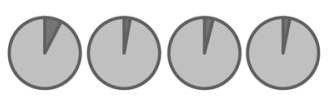

Avg. methylation $=5.7 \%$

Ezh2 ${ }^{N / \Delta} p 53^{N /}$

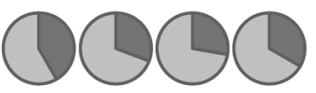

Avg. methylation $=29.5 \%$

Avg. methylation $=5.0 \%$

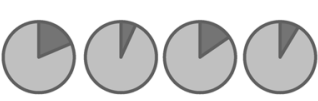

Avg. methylation $=12.9 \%$

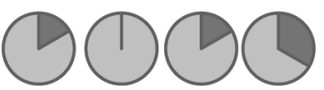

Avg. methylation $=20.3 \%$

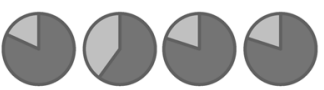

Avg. methylation $=79.8 \%$

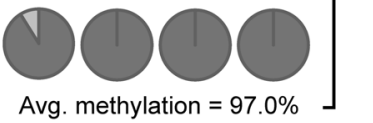


Figure 6. DNA hypermethylation propagated at promoters of critical genes for $\mathrm{T}$ cell developmental regulators following the loss of Ezh2 and p53. (A) Numbers of hypo- and hyper-DMRs at promoters in Ezh2 ${ }^{\Delta / \Delta}$ DN2 cells, $p 53^{\Delta / \Delta}$ DN2 cells, Ezh2 $2^{\Delta / \Delta} p 53^{\Delta / \Delta}$ DN2 cells at the preleukemic stage, and Ezh2 ${ }^{\Delta / \Delta} \mathrm{p} 3^{\Delta / \Delta}$ leukemic DN2 cells relative to WT DN2 cells. (B) Bars showing differences in the degree of methylation (\%) between Ezh2 $2^{\Delta / \Delta}$ p53 $3^{\Delta / \Delta}$ leukemic DN2 cells and WT DN2 cells. (C) GO biological process gene sets significantly enriched in hyper-DMR (red bars) and hypo-DMR (blue bars) genes in Ezh2 $2^{\Delta / \Delta} \mathrm{p} 53^{\Delta / \Delta}$ leukemic cells. (D) Scatter plots showing the relationship between the fold enrichment values (ChIP/input) of H3K27me3 (TSS $\pm 2.0 \mathrm{~kb}$ of RefSeq genes) in Ezh2 ${ }^{\Delta / \Delta} \mathrm{p} 53^{\Delta / \Delta}$ leukemic DN1 cells relative to WT DN1 cells and methylation differences in Ezh2 ${ }^{\Delta / \Delta} \mathrm{p} 53^{\Delta / \Delta}$ leukemic DN2 cells relative to WT DN2 cells. (E) Boxes showing the levels of DNA methylation (\%) of WT DN2 cells, Ezh2 ${ }^{\Delta / \Delta}$ DN2 cells, p53 ${ }^{\Delta / \Delta}$ DN2 cells, and Ezh2 $2^{\Delta / \Delta} \mathrm{p} 53^{\Delta / \Delta}$ DN2 cells at the preleukemic stage and Ezh2 $2^{\Delta / \Delta}$ p53 $3^{\Delta / \Delta}$ leukemic DN2 cells in RefSeq genes (All genes) and canonical PRC2 target genes (PRC2 targets). Data are shown as box-and-whiskers plots drawing 10 th to 90 th percentiles. ${ }^{* *} P<0.001$, Student's $t$ test. $(\mathbf{F})$ Scatter plots showing the relationship between expression changes in RefSeq genes in Ezh2 ${ }^{\Delta / \Delta} \mathrm{p} 53^{\Delta / \Delta}$ leukemic DN2 cells from those in WT DN2 cells and methylation differences in Ezh2 ${ }^{\Delta / \Delta} \mathrm{p} 53^{\Delta / \Delta}$ leukemic DN2 cells from those in WT DN2 cells. (G) ChIP-seq views of H3K27me3 and RRBS data of selected hyper-DMRs in WT DN2 cells, Ezh2 ${ }^{\Delta / \Delta}$ DN2 cells, p53 ${ }^{\Delta / \Delta}$ DN2 cells, and Ezh2 ${ }^{\Delta / \Delta} \mathrm{p} 53^{\Delta / \Delta}$ DN2 cells at the preleukemic stage and Ezh2 $2^{\Delta / \Delta} \mathrm{p} 53^{\Delta / \Delta}$ leukemic DN2 cells. Methylation differences between Ezh2 ${ }^{\Delta / \Delta} \mathrm{p} 53^{\Delta / \Delta}$ leukemic DN2 and WT DN2 cells that were significant are indicated. ${ }^{* * *} P<0.001$, Student's $t$ test.

methylation (38). Taken together, these findings show that Ezh2PRC2 may function to protect DNA methylation-prone CGI promoters from inappropriate DNA methylation in ETPs; however, once Ezh2-PCR2 is lost, the pathogenic epigenetic switch occurs at the gene loci of $\mathrm{T}$ cell development regulator genes, including tumor suppressor genes, such as Runx1, and promotes the transformation of ETPs.

While the loss of Ezh2-activated stem cell signature genes may confer an enhanced proliferative capacity to ETP-ALL cells, we also noted that RAS and JAK/STAT signaling pathways were both markedly activated in Ezh2p53-deficient leukemic cells from gene expression analyses. Patients with ETP-ALL frequently harbor activating mutations in genes involved in cytokine receptor and RAS signaling, such as IL7R, JAK1, NRAS, and KRAS (2). Gain-of function mutations in $I L 7 R$ lead to the ligand-independent activation of JAK1/STAT5 signaling (39), and the transduction of the $I L 7 R$ mutant in purified $p 19^{\text {Arf }}$-deficient thymocytes was sufficient to generate an ETP-ALL-like disease in mice (40). As described above, another mouse model utilizing the NRAS mutant and purified $p 19^{\text {Arf }}$-deficient thymocytes also showed enhanced Stat 3 phosphorylation upon the loss of Ezh2. Consistent with these findings, we found that a cytokine stimulation activated the JAK/STAT signal more strongly in Ezh2-deficient hematopoietic stem/progenitor cells than in WT cells (10). Human ETP-ALL cells show higher phosphorylation levels of STAT5 and STAT3 in response to an IL-7 stimulation than non-ETP T-ALL cells (41). In fact, the pharmacological inhibition of JAK/STAT signaling by the JAK2 inhibitor ruxolitinib resulted in significant antitumor effects in mice and xenograft models $(40,41)$. Although the mechanisms by which the inactivation of EZH2-PRC2 stimulates JAK/STAT signaling currently remain unknown, our mouse model also supports the importance of JAK/STAT signaling for the pathogenesis of ETP-ALL regardless of the presence of JAK/STAT pathway mutations, raising the therapeutic potential of the combination of ruxolitinib and DAC in ETP-ALL patients harboring PRC2 mutations. NOTCH1activating mutations were not frequently detected in ETP-ALL cells in patients or mice $(2,40)$. Correspondingly, the significant activation of Notch1 target genes was not observed in Ezh2p53deficient ETP-ALL cells in the present study (data not shown).

Collectively, we demonstrated that the combined deletion of Ezh2 and p53 in mice successfully recapitulated ETP-ALL, a subset of T-ALL showing poor clinical outcomes, for which novel therapies are needed. The loss of Ezh2 in ETPs induced the pathogenic epigenetic switch from H3K27me3 to DNA methylation of pivotal T cell development regulators, such as Runx1, and promoted the transformation of ETPs. Our results strongly support a pathogenic role for inactivating PRC2 mutations in ETP-ALL, thereby highlighting a tumor-suppressive function for EZH2-PRC2 in ETPs.

\section{Methods}

Mice and transplantation. Ezh2 conditional knockout $\left(E z h 2^{f / f l}\right)$ mice were described previously (42). These were crossed with Rosa26::CreERT2 mice (TaconicArtemis $\mathrm{GmbH}$ ) for conditional deletion. p53 conditional knockout $\left(p 53^{f / f}\right)$ mice were described previously (17). C57BL/6 mice congenic for the Ly5 locus (CD45.1) were purchased from Sankyo-Lab Service. A total of $5 \times 10^{6} \mathrm{BM}$ cells were intravenously injected into $8.5 \mathrm{~Gy}$ - or $9 \mathrm{~Gy}$-irradiated CD $45.1^{+}$mice. Four weeks after transplantation, $1 \mathrm{mg}$ tamoxifen (T5648, Sigma-Aldrich) was administered via an intraperitoneal injection for 5 consecutive days to completely delete the $p 53$ and Ezh2 alleles.

Plasmids and retroviral transduction. Retroviral transduction of pMYs-IRES-GFP, pMYs-RUNX1-IRES-GFP, and pMYs-Bcl11b-IRESGFP vectors into thymocytes was previously described $(9,43)$. Briefly, ETP-ALL cells were transduced with a vector on TSt-4/DLL1 stromal cells in the presence of SCF, IL-7, and Flt3L $(10 \mathrm{ng} / \mathrm{ml})$. In order to induce differentiation, transduced cells were cultured in the presence of SCF, IL-7, and Flt3L ( $2 \mathrm{ng} / \mathrm{ml})$ for 9 days. For knockdown of Dnmt, CS-H1-shRNA-EF-1 $\alpha$-EGFP vector expressing shRNA-directed Dnmt1, Dnmt3a, Dnmt3b, or Luciferase was used. Target sequences were as follows: shDnmt1 no. 1: ATCTATGGAAGGTGGTATTA; shDnmt1 no.2: TATATGAAGACCTGATCAAT; shDnmt3a no.1: GCACAACAGAGAAACCTAA; shDnmt3a no.2: GCAGACCAACATCGAATCCAT; sh Dnmt3b no.1: GCGGGTATGAGGAGTGCATTA; and $\operatorname{sh} D n m t 3 b$ no.2: CCAAGCGCCTCAAGACAAAT.

Flow cytometry and antibodies. Flow cytometry and cell sorting were performed by utilizing the following monoclonal antibodies: CD45.2 (clone 104), CD45.1 (clone A20), Gr1 (clone RB6-8C5), CD11b/Mac1 (clone M1/70), CD11c (clone N418), NK1.1 (clone PK136), Ter119 (catalog 116204), CD71 (clone R17217), CD127/ IL-7R $\alpha$ (clone A7R34), B220 (clone RA3-6B2), CD3e (clone 1452C11), CD4 (clone L3T4), CD8 $\alpha$ (clone 53-6.7), CD117/c-Kit (clone 2B8), Sca1 (clone D7), CD34 (clone MEC14.7), and Fc $\gamma$ RII-III (clone 93). The CD34 antibody was purchased from eBioscience. All others were from BioLegend. The lineage mixture solution contained biotin-conjugated anti-Gr1, anti-Mac1, anti-CD11c, anti-NK1.1, anti-B220, anti-CD3e, anti-CD4, anti-CD8 $\alpha$, and anti-Ter119 antibodies. All flow cytometry analyses and cell sorting were performed on FACSAriaII or FACSCantoII (BD). 
A

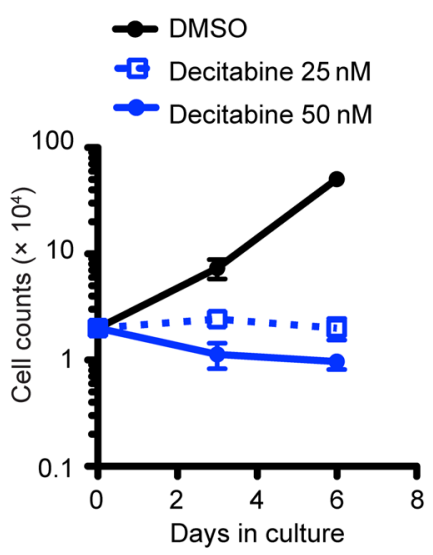

B
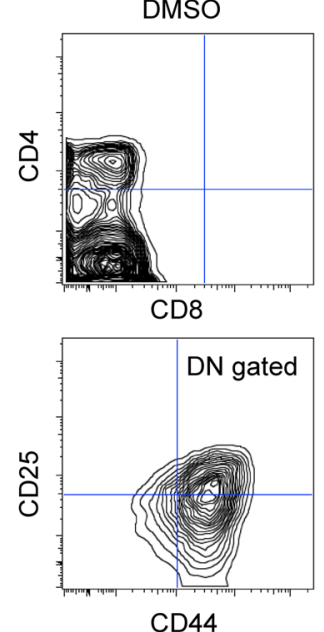

Decitabine $25 \mathrm{nM}$

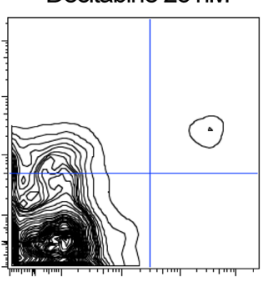

CD8

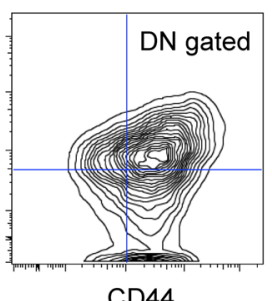

CD44
Decitabine $50 \mathrm{nM}$

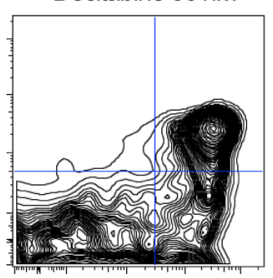

CD8

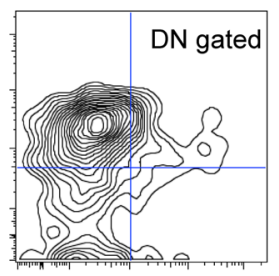

CD44

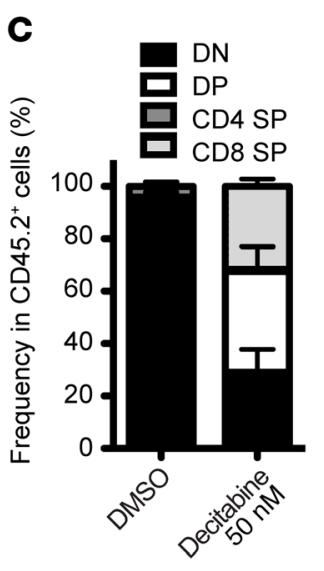

$\mathbf{F}$

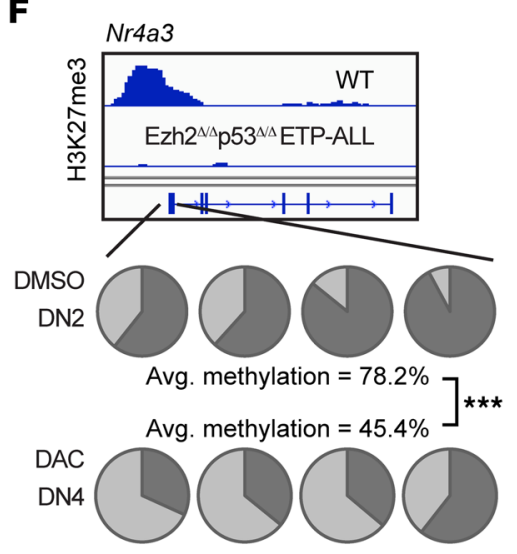

Runx1

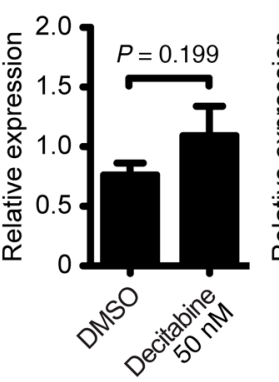

Nr4a3

$B c / 11 b$

E
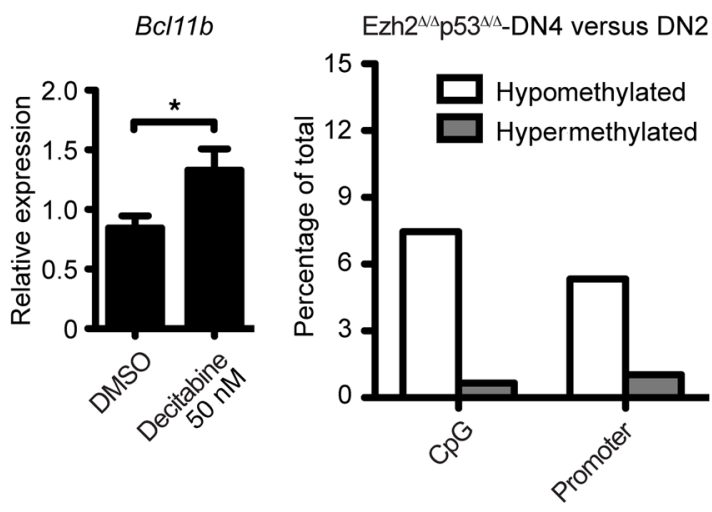

G

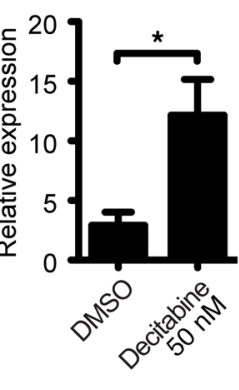

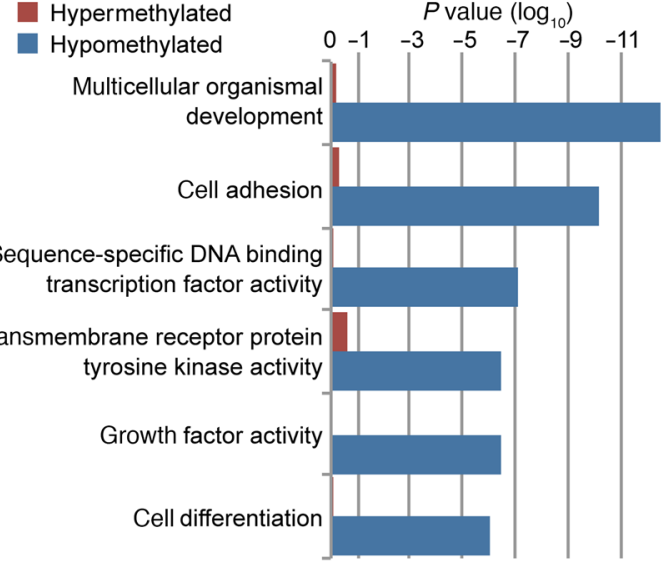

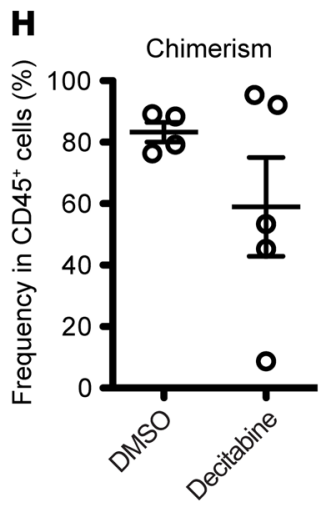

I

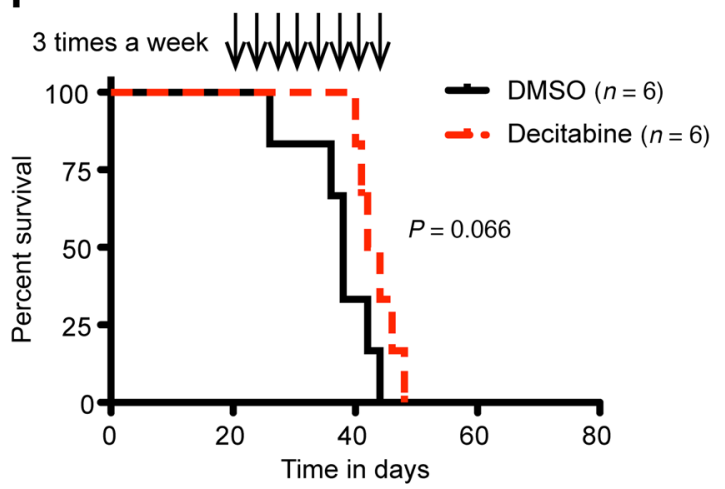


Figure 7. Aberrant DNA hypermethylation sensitized ETP-ALL cells to a DNA hypomethylating agent. (A) Total cell counts of control (black line) and DAC-treated ( $25 \mathrm{nM}$, blue broken line; $50 \mathrm{nM}$, blue line) Ezh2 $2^{\Delta / \Delta}$ p53 $3^{\Delta / \Delta}$ ETP-ALL cells monitored for 6 days in the culture $(n=3)$. Data are representative of 3 independent experiments. (B) Representative flow cytometric profiles of CD4 and CD8 expression (top) and CD44 and CD25 expression in CD4-CD8- DN cells (bottom) on day 6 of the culture in A. (C) Proportions of CD4+CD8 ${ }^{+}$DP cells, CD4 SP cells, CD8 SP cells, and CD4-CD8DN cells in control and DAC-treated $(50 \mathrm{nM})$ cultures on day $6(n=4-5)$. (D) Quantitative RT-PCR analysis of the expression of Runx1, Bc/11b, and Nr4a3 in purified DN2 cells harvested from control and ETP-ALL cells treated with $50 \mathrm{nM}$ DAC on day 3 of the culture $(n=6-9)$. (E) Numbers of hypo- and hyper-DMRs at CGIs and promoters in DAC-induced Ezh2 $2^{\Delta / \Delta} \mathrm{p} 53^{\Delta / \Delta}$ leukemic DN4 cells relative to DMSO-induced Ezh2 $2^{\Delta / \Delta} \mathrm{p} 53^{\Delta / \Delta}$ leukemic DN2 cells. (F) ChIP-seq view of H3K27me3 levels at the Nr4a3 locus in WT and Ezh2 $2^{\Delta / \Delta}$ p53 $3^{\Delta / \Delta}$ leukemic DN1 cells and RRBS data of DNA methylation at the $\mathrm{Nr} 4 a 3$ locus in Ezh2 ${ }^{\Delta / \Delta} \mathrm{p}^{2} 3^{\Delta / \Delta}$ leukemic DN2 cells and DAC-induced Ezh2 ${ }^{\Delta / \Delta}$ p53 $3^{\Delta / \Delta}$ leukemic DN4 cells. ${ }^{* *} P<0.001$, Student's $t$ test. (C) GO biological process gene sets enriched in hyper-DMRs (red bars) and hypo-DMRs (blue bars). (H) Chimerism of CD45.2+ cells in the PB cells of DMSO-treated and DAC-treated ( $0.2 \mathrm{mg} / \mathrm{kg}, 3$ times a week) Ezh2 $2^{\Delta / \Delta} \mathrm{p} 53^{\Delta / \Delta}$ leukemic mice 3 weeks after the completion of the treatment $(n=4-5)$. (I) Longer median survival of DAC-treated Ezh2 $2^{\Delta / \Delta} \mathrm{p} 53^{\Delta / \Delta}$ leukemic mice compared with DMSO-treated mice $(n=6)$. Representative data of the 2 independent experiments are shown. $P$ value is by log-rank test.

Western blotting. The antibodies used for Western blotting were as follows: anti-H3K27me3 (07449, Merck Millipore) and anti-H3 (ab1791, Abcam).

Quantitative RT-PCR. Quantitative RT-PCR was performed on a StepOnePlus Real-Time PCR System (Life Technologies) by using SYBR Premix Ex TaqII (Takara) or FastStart Universal Probe Master (Roche) with the Universal ProbeLibrary (Roche). Primer sequences with Universal ProbeLibrary (Roche) numbers are as follows: Ezh2 (forward, CCAGACTGGTGAAGAGTTGTTTT; reverse, CAAGGGATTTCCATTTCTCG; no. 105); 553 (forward, ATGCCCATGCTACAGAGGAG; reverse, AGACTGGCCCTTCTTGGTCT; no. 94); p16 $6^{\text {Ink4a }}$ (forward, AATCTCCGCGAGGAAAGC; reverse, GTCTGCAGCGGACTCCAT; no. 91); p19ARF (forward, GGGTTTTCTTGGTGAAGTTCG; reverse, TTGCCCATCATCATCACCT; no. 106); Nr4a3 (forward, GTGTCGGGATGGTTAAGGAA; reverse, CCTGTTGTAGTGGGCTCTTTG; no. 97); Runx1 (forward, CTCCGTGCTACCCACTCACT; reverse, ATGACGGTGACCAGAGTGC; no. 77); Bcl11b (forward, TGGATGCCAGTGTGAGTTGT; reverse, GCTGCTTGCATGTTGTGC; no. 97); Ptcra (forward, TCAGGTGTCAGGCTCTACCA; reverse, GCCTTCCATCTACCAGCAGT; no. 106); Hlf (forward, CTACGGCGTGCTCAGGTC; reverse, TCCTTTGTCTTTTTCTTTACTAAATGC; no. 67); Hoxa10 (forward, CCTTCAGAAAACAGTAAAGCTTCG; reverse, AAGGGCAGCGTTTCTTCC; no. 100); Dnmt1 (forward, CATGGTCTTCCCССАСTCTCTTG; reverse, ACAGAACAGCTCTGAACGAGACC); Dnmt3a (forward, GAAACTAAGGTGCTGGGGACTGG; reverse, CCTTAGTTCTGTGACCCTGGTGC); Dnmt3b (forward, TTGTGCCAGACCTTGGAAACCTC; reverse, AAAGAAGGCCCTGGATTTGGTCC); and Gapdh (forward, ATGACATCAAGAAGGTGGTGAAG; reverse, TCCTTGGAGGCCATGTAGG). All data are presented as relative expression levels normalized to Gapdh expression.

Microarray and data analyses. Total RNA was extracted from approximately $1 \times 10^{5}$ pooled cells from 5 to 10 mice using an RNeasy Plus Mini Kit (QIAGEN). Twenty nanograms of total RNA was mixed with spike-in controls using an Agilent One Color Spike Mix Kit, amplified, and labeled with Cyanine 3 using a Low Input Quick Amp Labeling Kit (Agilent) according to the manufacturer's instructions. A microarray analysis using a SurePrint G3 Mouse GE Microarray 8x60K Kit (Agilent) was performed according to the manufacturer's instructions. Hierarchical clustering and principal component analyses based on total gene expression were performed using WebMeV (Multiple Experiment Viewer) software.

RRBS. RRBS was performed as previously described $(9,24)$.

ChIP sequencing. ChIP assays were performed as previously described (8). Briefly, $1 \times 10^{5}$ pooled DN1 cells were used for each immunoprecipitation reaction with an anti-H3K27me3 (Merck Millipore, 07449) antibody.

Sequencing data analysis. The ChIP-seq signal was quantified as the total number of reads per million. In order to evaluate the histone modification mark of each gene, normalized tag numbers in the region from $2 \mathrm{~kb}$ upstream to $2 \mathrm{~kb}$ downstream of the TSS were counted and divided by the tag number of the corresponding input. The reads per kilobase of transcripts per million mapped reads (RPKM) values of the sequenced reads were calculated every 5,000 bp bin with a shifting size of 500 bp using bedtools to be visualized with the Integrative Genomics Viewer (IGV) genome browser. The read numbers of the immunoprecipitated samples were then normalized by subtracting the RPKM values of the input samples in each bin and converted to a BigWig file using the wigToBigWig tool. The supercomputing resource was provided by the Human Genome Center, Institute of Medical Science, University of Tokyo (http://sc.hgc.jp/shirokane.html).

PCR analysis of TCR- $\beta$ gene rearrangement. Genomic DNA was extracted from approximately $1 \times 10^{5} \mathrm{DN} 2$ cells. Extracted DNA was amplified by PCR with primers of D $\beta-1.1$, extension GAGGAGCAGCTTATCTGGTG, and J $\beta-1.7$, extension AAGGGACGACTCTGTCTTAC, by using T100 Thermal Cycler (Bio-Rad). A PCR product of the first amplification was subjected to a second PCR with nested primers of D $\beta-1.1$, internal GGTAGACCTATGGGAGGGC, and J $\beta-1.7$, internal ACCATGGTCATCCAACACAG, as previously described (44).

Statistics. All statistical tests were performed using GraphPad Prism version 7 (GraphPad Software). The significance of differences was measured by an unpaired 2-tailed Student's $t$ test or Mann-Whitney nonparametric test. A $P$ value of less than 0.05 was considered significant.

Study approval. All animal studies were reviewed and approved by the Review Boards for Animal Experiments of Chiba University (approval ID: 30-56) and Kumamoto University (approval ID: A 30-006).

Accession codes. ChIP sequencing and RRBS data were deposited in the DNA Data Bank of Japan (DDBJ PRJDB5585 and PRJDB6937). All original microarray data were deposited in the NCBI's Gene Expression Omnibus database (GEO GSE95655).

\section{Author contributions}

$\mathrm{CW}$ performed experiments and analyzed results. $\mathrm{MO}$ analyzed results. DS, HM, SK, KA, YNT, SK, JM, MMK, TNY, and JB performed experiments. TN provided reagents. AK analyzed results. $\mathrm{AI}$ and GS designed the research, performed experiments, analyzed results, and wrote the manuscript.

\section{Acknowledgments}

The authors thank the members of the Iwama Laboratory for their discussions during the preparation of this manuscript. The authors thank Haruhiko Koseki and Anton Berns for kindly pro- 
viding Ezh2 and p53 conditional knockout mice, respectively, and Hiroshi Kawamoto for kindly providing TSt-4/DLL4 cells. The supercomputing resource was provided by the Human Genome Center (Institute of Medical Science, University of Tokyo). This work was supported in part by Grants-in-Aid for Scientific Research (nos. 15H02544, 25130702, 26461396, and 16KT0113), Scientific Research on Innovative Areas Cell Fate (no. 22118004) and Stem Cell Aging and Disease (no. 26115002) from the Ministry of Education, Culture, Sports, Science and
Technology, Japan, a Program of Higher-Level Talents of Inner Mongolia University award (to Changshan Wang), and the National Natural Science Foundation of China (no. 81660024).

Address correspondence to: Goro Sashida, 2-2-1 Honjo, Chuo-ku, Kumamoto 860-0811, Japan. Phone: 81.96.373.6827; Email: sashidag@kumamoto-u.ac.jp. Or to: Atsushi Iwama, 4-6-1 Shirokanedai, Minato-ku, Tokyo 108-8639, Japan. Phone: 81.3.6409.2180; Email: 03aiwama@ims.u-tokyo.ac.jp.
1. Coustan-Smith E, et al. Early T-cell precursor leukaemia: a subtype of very high-risk acute lymphoblastic leukaemia. Lancet Oncol. 2009;10(2):147-156.

2. Zhang J, et al. The genetic basis of early T-cell precursor acute lymphoblastic leukaemia. Nature. 2012;481(7380):157-163.

3. Mochizuki-Kashio M, et al. Dependency on the polycomb gene Ezh2 distinguishes fetal from adult hematopoietic stem cells. Blood. 2011;118(25):6553-6561.

4. Xie H, et al. Polycomb repressive complex 2 regulates normal hematopoietic stem cell function in a developmental-stage-specific manner. Cell Stem Cell. 2014;14(1):68-80.

5. Shih AH, Abdel-Wahab O, Patel JP, Levine RL. The role of mutations in epigenetic regulators in myeloid malignancies. Nat Rev Cancer. 2012;12(9):599-612.

6. Iwama A. Polycomb repressive complexes in hematological malignancies. Blood. 2017;130(1):23-29.

7. Ntziachristos $P$, et al. Genetic inactivation of the polycomb repressive complex 2 in $\mathrm{T}$ cell acute lymphoblastic leukemia. Nat Med. 2012;18(2):298-301.

8. Muto T, et al. Concurrent loss of Ezh2 and Tet2 cooperates in the pathogenesis of myelodysplastic disorders. JExp Med. 2013;210(12):2627-2639.

9. Sashida G, et al. Ezh2 loss promotes development of myelodysplastic syndrome but attenuates its predisposition to leukaemic transformation. Nat Commun. 2014;5:4177.

10. Sashida G, et al. The loss of Ezh2 drives the pathogenesis of myelofibrosis and sensitizes tumor-initiating cells to bromodomain inhibition. J Exp Med. 2016;213(8):1459-1477.

11. Mochizuki-Kashio M, et al. Ezh2 loss in hematopoietic stem cells predisposes mice to develop heterogeneous malignancies in an Ezh1-dependent manner. Blood. 2015;126(10):1172-1183.

12. Simon C, et al. A key role for EZH2 and associated genes in mouse and human adult T-cell acute leukemia. Genes Dev. 2012;26(7):651-656.

13. Danis E, et al. Ezh2 controls an early hematopoietic program and growth and survival signaling in early T cell precursor acute lymphoblastic leukemia. Cell Rep. 2016;14(8):1953-1965.

14. Donehower LA, et al. Mice deficient for p53 are developmentally normal but susceptible to spontaneous tumours. Nature. 1992;356(6366):215-221.

15. Grossmann V, et al. The molecular profile of adult T-cell acute lymphoblastic leukemia: mutations in RUNX1 and DNMT3A are associated with poor prognosis in T-ALL. Genes Chromosomes Cancer. 2013;52(4):410-422.

16. Seki M, et al. Recurrent SPI1 (PU.1) fusions in high-risk pediatric $\mathrm{T}$ cell acute lymphoblastic leukemia. Nat Genet. 2017;49(8):1274-1281.

17. Jonkers J, Meuwissen R, van der Gulden $H$, Peterse H, van der Valk M, Berns A. Synergistic tumor suppressor activity of BRCA2 and p53 in a conditional mouse model for breast cancer. Nat Genet. 2001;29(4):418-425.

18. Cheng LE, Chan FK, Cado D, Winoto A. Functional redundancy of the Nur77 and Nor-1 orphan steroid receptors in T-cell apoptosis. EMBO J. 1997;16(8):1865-1875

19. Belyaev NN, Biró J, Athanasakis D, FernandezReyes D, Potocnik AJ. Global transcriptional analysis of primitive thymocytes reveals accelerated dynamics of $\mathrm{T}$ cell specification in fetal stages. Immunogenetics. 2012;64(8):591-604.

20. Ikawa T, et al. An essential developmental checkpoint for production of the T cell lineage. Science. 2010;329(5987):93-96.

21. Li L, Leid M, Rothenberg EV. An early T cell lineage commitment checkpoint dependent on the transcription factor Bcl11b. Science. 2010;329(5987):89-93.

22. Grossmann V, et al. Prognostic relevance of RUNX1 mutations in T-cell acute lymphoblastic leukemia. Haematologica. 2011;96(12):1874-1877.

23. Chambers SM, et al. Hematopoietic fingerprints: an expression database of stem cells and their progeny. Cell Stem Cell. 2007;1(5):578-591.

24. Hasegawa $\mathrm{N}$, et al. Impact of combinatorial dysfunctions of Tet2 and Ezh2 on the epigenome in the pathogenesis of myelodysplastic syndrome. Leukemia. 2017;31(4):861-871.

25. Lister R, et al. Human DNA methylomes at base resolution show widespread epigenomic differences. Nature. 2009;462(7271):315-322.

26. Challen GA, et al. Dnmt3a is essential for hematopoietic stem cell differentiation. Nat Genet. 2011;44(1):23-31.

27. Morin RD, et al. Somatic mutations altering EZH2 (Tyr641) in follicular and diffuse large B-cell lymphomas of germinal-center origin. Nat Genet. 2010;42(2):181-185.

28. Béguelin W, et al. EZH2 is required for germinal center formation and somatic EZH2 mutations promote lymphoid transformation. Cancer Cell. 2013;23(5):677-692.

29. Tanaka S, et al. Ezh2 augments leukemogenicity by reinforcing differentiation blockage in acute myeloid leukemia. Blood. 2012;120(5):1107-1117.

30. Neff T, et al. Polycomb repressive complex 2 is required for MLL-AF9 leukemia. Proc Natl Acad
Sci U S A. 2012;109(13):5028-5033.

31. Souroullas GP, et al. An oncogenic Ezh2 mutation induces tumors through global redistribution of histone 3 lysine 27 trimethylation. Nat Med. 2016;22(6):632-640.

32. $\mathrm{Su} \mathrm{IH}$, et al. Polycomb group protein ezh 2 controls actin polymerization and cell signaling. Cell. 2005;121(3):425-436.

33. Yui MA, Rothenberg EV. Developmental gene networks: a triathlon on the course to T cell identity. Nat Rev Immunol. 2014;14(8):529-545.

34. Rasmussen KD, Helin K. Role of TET enzymes in DNA methylation, development, and cancer. Genes Dev. 2016;30(7):733-750.

35. Manzo M, Wirz J, Ambrosi C, Villaseñor R, Roschitzki B, Baubec T. Isoform-specific localization of DNMT3A regulates DNA methylation fidelity at bivalent $\mathrm{CpG}$ islands. $E M B O J$. 2017;36(23):3421-3434.

36. Ohm JE, et al. A stem cell-like chromatin pattern may predispose tumor suppressor genes to DNA hypermethylation and heritable silencing. Nat Genet. 2007;39(2):237-242.

37. Maegawa S, et al. Age-related epigenetic drift in the pathogenesis of MDS and AML. Genome Res. 2014;24(4):580-591.

38. Boulard M, Edwards JR, Bestor TH. FBXL10 protects Polycomb-bound genes from hypermethylation. Nat Genet. 2015;47(5):479-485.

39. Zenatti PP, et al. Oncogenic IL7R gain-offunction mutations in childhood T-cell acute lymphoblastic leukemia. Nat Genet. 2011;43(10):932-939.

40. Treanor LM, et al. Interleukin-7 receptor mutants initiate early $\mathrm{T}$ cell precursor leukemia in murine thymocyte progenitors with multipotent potential. J Exp Med. 2014;211(4):701-713.

41. Maude SL, et al. Efficacy of JAK/STAT pathway inhibition in murine xenograft models of early T-cell precursor (ETP) acute lymphoblastic leukemia. Blood. 2015;125(11):1759-1767.

42. Hirabayashi Y, et al. Polycomb limits the neurogenic competence of neural precursor cells to promote astrogenic fate transition. Neuron. 2009;63(5):600-613.

43. Mishima Y, et al. Histone acetylation mediated by Brd1 is crucial for $\mathrm{Cd} 8$ gene activation during early thymocyte development. Nat Commun. 2014;5:5872.

44. King AG, Kondo M, Scherer DC, Weissman IL. Lineage infidelity in myeloid cells with TCR gene rearrangement: a latent developmental potential of proT cells revealed by ectopic cytokine receptor signaling. Proc Natl Acad Sci U S A. 2002;99(7):4508-4513. 